\title{
Journal of Bionic Engineering \\ Growth of TiO2 Nanotube on Titanium Substrate to Enhance its Biotribological Performance and Biocorrosion Resistance \\ --Manuscript Draft--
}

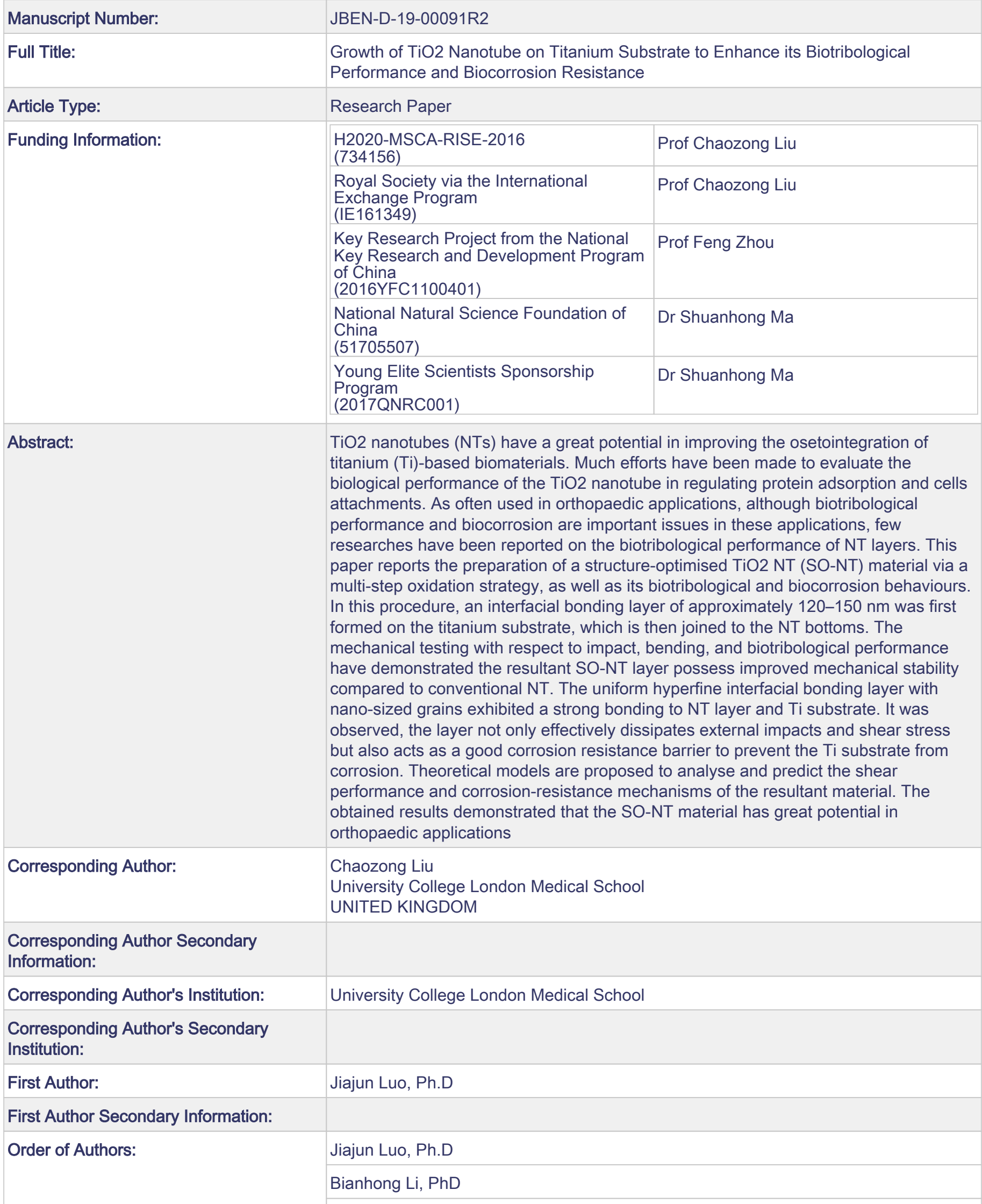


Sara Ajami, PhD

Shuanhong $\mathrm{Ma}$

Feng Zhou, PhD

Chaozong Liu, PhD

Order of Authors Secondary Information:

Author Comments:

Response to Reviewers:

Dear Prof Da He,

Re: JBEN-D-19-00091R1

Title: Bionic Engineering of TiO2 Nanotube Growth on Titanium Substrate to Enhance its Biotribological Performance and Biocorrosion Resistance

Thank you for your MESSAGE OF 1 Nov 2019 in which you provided Dr Dan Zhang's comments on our above titled paper. In accordance with the comments made by the Dr. Zhang, we have made the following amendments to the manuscript and the changes have been highlighted in the revised manuscript:

1.Fig. S5 should be cited before Figs. S6 and S7.

We have adjusted the sequence of the Figures in the supporting information according to the reporting sequence in the context. Accordingly, we have also changed the Figure number to reflect this change. Please reference to the revised Supporting Information which highlighted the changes.

2.To save cost, unless necessary, we do not publish color figures. So we would suggest you to revise Fig. 3 as white/black figure with different line styles.

We have changed Figure 3 to white/black image.

Please find the revised version of the paper (Ref. No. JBEN-D-19-00091) which we trust it is now in a form appropriate for publication in Journal of Bionic Engineering. Should you have any further questions regarding the paper, please do not hesitate to contact me.

Yours Sincerely,

Jiajun Luo \& Chaozong Liu

\section{Suggested Reviewers:}

Dangsheng Xiong, PhD

Professor, Nanjing University of Science \& Technology

xiongds@163.com

Professor Xiong has expertise in biotribology study

Xiaodan Sun, PhD

Associate Professor, Tsinghua University

sunxiaodan@tsinghua.edu.cn

Dr Xiaodan Sun is an expert in Biomaterials processing.

Ling Wang, PhD

Associate Professor, Xi'an Jiaotong University

menlwang@xjtu.edu.cn

Dr Ling Wang is an expert in biotribology testing and has expertise in FE modelling 


\title{
Growth of $\mathrm{TiO}_{2}$ Nanotube on Titanium Substrate to Enhance its Biotribological Performance and Biocorrosion Resistance
}

\author{
Jiajun Luo ${ }^{1}$, Bianhong $\mathrm{Li}^{1,3}$, Sara Ajami ${ }^{1}$, Shuanhong Ma ${ }^{2 *}$, Feng Zhou², Chaozong Liu ${ }^{1 *}$ \\ 1. Institute of Orthopaedic \& Musculoskeletal Science, Division of Surgery \& Interventional Science, University \\ College London, Royal National Orthopaedic Hospital, Stanmore, HA7 4LP, United Kingdom \\ 2. State Key Laboratory of Solid Lubrication, Lanzhou Institute of Chemical Physics, Chinese Academy of Sciences, \\ Lanzhou, 730000, China \\ 3. School of Mechatronical Engineering, Beijing Institute of Technology, Beijing City, 100081, China
}

* Corresponding authors

Prof Chaozong Liu

UCL IOMS, University College London, Royal National Orthopaedic Hospital, Stanmore HA7 4LP, UK

E-mail: Chaozong.Liu@ucl.ac.uk

Dr Shuanhong Ma

State Key Laboratory of Solid Lubrication, Lanzhou Institute of Chemical Physics, Chinese Academy of Sciences, Lanzhou, 730000, China

E-mail: mashuanhong@licp.cas.cn 


\begin{abstract}
TiO2 nanotubes (NTs) have a great potential in improving the osetointegration of titanium (Ti)-based biomaterials. Much efforts have been made to evaluate the biological performance of the $\mathrm{TiO}_{2}$ nanotube in regulating protein adsorption and cells attachments. As often used in orthopaedic applications, although biotribological performance and biocorrosion are important issues in these applications, few researches have been reported on the biotribological performance of NT layers. This paper reports the preparation of a structure-optimised TiO2 NT (SO-NT) material via a multi-step oxidation strategy, as well as its biotribological and biocorrosion behaviours. In this procedure, an interfacial bonding layer of approximately 120-150 nm was first formed on the titanium substrate, which is then joined to the NT bottoms. The mechanical testing with respect to impact, bending, and biotribological performance have demonstrated the resultant SO-NT layer possess improved mechanical stability compared to conventional NT. The uniform hyperfine interfacial bonding layer with nano-sized grains exhibited a strong bonding to NT layer and Ti substrate. It was observed, the layer not only effectively dissipates external impacts and shear stress but also acts as a good corrosion resistance barrier to prevent the Ti substrate from corrosion. Theoretical models are proposed to analyse and predict the shear performance and corrosion-resistance mechanisms of the resultant material. The obtained results demonstrated that the SO-NT material has great potential in orthopaedic applications
\end{abstract}

Keywords: $\mathrm{TiO}_{2}$ nanotubes; tribology; titanium implant; surface modification; biomaterials;

\title{
1 Introduction
}

Over the past few decades, self-organised $\mathrm{TiO}_{2}$ nanotubes (NT) fabricated via anodisation have attracted attention. Owing to their specific morphology and outstanding physiochemical properties, it has a great potential for biomedical implants [1-3]. Furthermore, NTs have three dimensional nanostructure, can mimic natural bone from microscale to nanoscale [4]. One of the important previous studies has proved natural bone contains three structures in scales, non-collageneous organic proteins, collagen and assembled mineral crystals at nanoscale; osteons and lamellae at microscale; as well as bone in macroscale [5]. Nanostructured biomedical implant surface has gained attraction 
as it can provide a biologically inspiring topography for protein adsorption and cell interactions due to its nanostructure with mimetic natural tissues [6-9].

The titanium nanotubes can remarkably promote osteointegration and biofunctions in vivo, which has been widely proven in animal model such as rabbits, mini pigs, and rats models [10-14]. However, less attention has been devoted to the biotribological performance of NT [15]. According to clinical reports, osteointegration and in vivo performance are strongly affected by the wear behaviour of the implant surface. Wear particles generated at a bone implant interface activate macrophages, thereby initiating a cytokine signal cascade, which leads to osteoclastogenesis and bone resorption [16, 17]. Apart from the biotribological performance, corrosion and dissolution of the metallic implant can release metallic ions into the body leading to adverse biological reactions and mechanical failures [18]. It is well known that when a titanium (Ti) implant is inserted in a human body, it is surrounded by blood-rich tissue, the body fluid can lead to a synergistic degradation along with micro-motions at the bone-implant interface [19]. Small micro-motions may worsen during long term implant use, which can progress to greater loosening. Limiting the micro-motion and minimizing wear particles delaminating off from implant is a vital factor to osteointegration.

As orthopaedic implant success can be undermined by two leading causes, aseptic loosening and infection, implant coating are required to achieve inhibit microbial cells while still promoting the osteointegration. Many studies have focused on biofunctional NT to enhance their antibacterial activity or interactions with cells [20-23]. However, the biotribological and biocorrosion performances of NT, which play a key role in the long-term success of an implantation, are often missed. Conventional NT exhibited a weak mechanical stability, which limits their orthopaedic usage for bone implant [24-26]. To improve the mechanical stability, the sample can be annealed. Xiong et al [24] an- 
nealed NT at $500{ }^{\circ} \mathrm{C}$ and found more formed Ti-O bonds at the NT layer-Ti interface than that of pristine NT, which improved the adhesion. Correspondingly, a model for stress accumulation was proposed to explain the detaching mechanism of NT films, which tend to lift off from Ti substrates when the accumulated stress reaches a critical value [25]. Moreover, Wang et al. built micro-nano surface structures on a Ti substrate to enhance the mechanical stability of the NT layer on the Ti substrate [26]. Recently, additional anodisation method has attempted by Yu by create a compact layer that enhanced the NT adhesion strength [27]. Further, two steps anodisation can also improves hexagonal self-ordering level of NT, and reduce the variation of average pore diameter. Thus, further optimised modification conversional NT by anodisation is an effective strategy to obtain more ordered and enhanced properties of NT [28].

Because of the weak mechanical stability, the study of biotribological performance of NT is commonly disregarded. Furthermore, few studies were reported on the biocorrosion performance of NT. For example, Alves et al. [29] investigated NT dental implants based on Ca-, P-, and Zn-rich biofunctional NT using the reverse polarisation methodology. They revealed a tribo-electrochemical behaviour in artificial saliva. This study focused on preparing a rigid NT material and systematically investigating its biotribological and biocorrosion performances.

Structure-optimised $\mathrm{TiO}_{2}$ NT (SO-NT) were produced by introducing a 120-150 nm interfacial bonding layer to the NT layer-Ti substrate interface via multiple anodization. This paper reported the biotribological and biocorrosion performances of the resultant SO-NT layer. Furthermore, the enhanced wear resistance along with a theoretical model were discussed in detail.

\section{Materials and methods}




\subsection{Fabrication of Structure-Optimised $\mathrm{TiO}_{2}$ Nanotubes (SO-NT) and Conventional $\mathrm{TiO}_{2} \mathrm{Nanotubes}$}

(NT)

A Ti foil (99.5\%; thickness of $0.15 \mathrm{~mm}$ ) was sequentially cleaned in acetone, ethanol, and deionised (DI) water, and then dried in air. The SO-NT were prepared via a multiple-anodisation process, as illustrated in Figure 1. First, the Ti substrate was anodised in ethylene glycol with $0.5 \mathrm{wt} \% \mathrm{NH}_{4} \mathrm{~F}$ for $30 \mathrm{~min}$ in an anodisation bath (under constant $60 \mathrm{~V}(\mathrm{DC})$ ) to obtain conventional NT. To fabricate SO-NT, the formed surficial NT layer was lifted off through ultrasonic cleaning in DI water. This create a bowl-like print on the Ti surface. The sample was then anodised in an ethylene glycol solution containing 3 wt\% $\mathrm{H}_{3} \mathrm{PO}_{4}$ for 120 s to generate a $100 \mathrm{~nm}$ grain bonding layer. Afterward, anodisation in an ethylene glycol solution containing $0.5 \mathrm{wt} \% \mathrm{NH}_{4} \mathrm{~F}$ for $60 \mathrm{~min}$ under $60 \mathrm{~V}$ and anodisation in an ethylene glycol solution containing $3 \mathrm{wt} \% \mathrm{H}_{3} \mathrm{PO}_{4}$ followed. Finally, after rinsing with DI water and drying with an $\mathrm{N}_{2}$ stream, the SO-NT sample was obtained. Additional reference NT were fabricated using the same procedure as for SO-NT but without anodisation in 3 wt $\% \mathrm{H}_{3} \mathrm{PO}_{4}$.

\subsection{Physiochemical Characterisation of NT and SO-NT}

\subsubsection{Nanostructure analysis}

The morphologies of NT and SO-NT were examined with a high-resolution scanning electron microscope (HR-SEM) JSM-6701F (JEOL company, Japan) using a constant imaging voltage of $5 \mathrm{kV}$ with 8.0-8.5 $\mathrm{mm}$ working distance. For SEM examination, the samples were rinsed with distilled water, and cleaned in alcohol for each $10 \mathrm{~min}$ and dried with an $\mathrm{N}_{2}$ stream, respectively. The sample were coated with a thin film of gold to enhance the conductivity.

\subsubsection{X-ray photoelectron spectroscopy}


X-ray photoelectron spectroscopy (XPS) (PHI-5702, Physical Electronics, USA) was used to analyse the chemical composition of the surface layers of both specimens.

\subsubsection{Energy dispersive spectroscopy (EDS)}

The elemental distribution was measured by EDS mapping, both NT and SO-NT specimens were analyzed by SEM-EDS (Phenom ProX G5) .

\subsubsection{Wettability}

The specimen wettability was carried out by measuring water droplet contact angle by (DSA-100 optical contact angle meter, Kruss Company, Ltd., Germany)meter at room temperature. Each five Ti, NT and SO-NT specimens were measured and statistic analyzed.

\subsection{Biotribology and Biocorrosion}

\subsubsection{Mechanical stability}

Impact, foil-bending/-twisting, shearing, ultrasonic, and biotribological tests were conducted to evaluate the mechanical stability of the resultant surfaces. The bending test was carried out first to evaluate the combination strength of the NT layer on the Ti substrate. Considering that each twist can lead to a different bending force, it was necessary to bend both NT and SO-NT. Both were fabricated on a Ti foil, which was bent by $150^{\circ}$ to compare the strengths of the attached NT layers. As the NT layers covered both sides of the Ti foil, one surface was under tension and the other under compression. Hence, the adhesion strength could be evaluated. Next, the mechanical strength was determined under more harsh conditions: The samples were continuously impacted with a metal hammer. Moreover, a falling-ball experiment in which a $1 \mathrm{~kg}$ steel ball (10 $\mathrm{mm}$ in diameter) was dropped from a height of $500 \mathrm{~mm}$ to samples was conducted to investigate the impact resistance. 
The crashed topography of samples were examined by optical observation and SEM. Furthermore, the samples were put into a ultrasonic cleaner with DI water (see supporting information) under vibration $(50 \mathrm{kHz}$ ) for different durations to compare the peel-off rates of SO-NT and NT when subjected to water friction. The biotribological performance was evaluated via a ball-on-disk reciprocating tribometer (CSM Instruments, USA) with a PTFE ball (diameter of $10 \mathrm{~mm}$ ) as counterpart in a 0.1 $\mathrm{g} / \mathrm{ml}$ bovine serum albumin lubricant under a $2 \mathrm{~N}$ load at $1 \mathrm{~Hz}$ for $120 \mathrm{~min}$. The coefficient of the friction evolution during the sliding test was recorded via the tribometer. The wear tracks were analysed and compared after $120 \mathrm{~min}$. The substrate-independent elastic modulus of NT and SO-NT were measured via nanoindentation with a Berkovich indenter at an indentation of below $300 \mathrm{~nm}$ (less than $10 \%$ of NT layer thickness). The effects of layer and substrate of SO-NT were neglected. The elastic modulus of NT and SO-NT $\left(\mathrm{E}_{\mathrm{N}}\right)$ were calculated as follows:

$$
\frac{1}{E_{\mathrm{r}}}=\frac{1-v_{\mathrm{N}}^{2}}{\mathrm{E}_{\mathrm{N}}}+\frac{1-v_{\mathrm{d}}^{2}}{\mathrm{E}_{\mathrm{d}}}
$$

where $E_{d}$ (elastic modulus of diamond tip) $=1194 \mathrm{GPa}, v_{d}$ (Poisson ratio of diamond tip) $=0.07$, $v_{\mathrm{N}}($ Poisson ratio of $\mathrm{Ti})=0.37$, and $\mathrm{E}_{\mathrm{r}}$ (relative elastic modulus) were measured via indentation. Three different NT and SO-NT sample areas were randomly selected for the modulus measurements to determine statistical results (see supporting information). The NT and SO-NT wear track morphologies were analysed with an SEM (JSM-5600LV, JEOL company, Japan) after the biotribology tests.

A six-stations pin-on-disk procedure was conducted to determine the volume loss and wear resistance of the NT and SO-NT: A stainless-steel pin with spherical end was applied to the NT and SO-NT disks with reciprocation and five-degree rotation (Figure S1). Three NT and three SO-NT disks were randomly distributed in the six stations. The parameters of the wear tests are listed in Table S1. 
By employing the Hertz contact theory, ASTM G99, and the contact stress, and volume loss were calculated. The profiles and depths of the wear tracks were measured employing TESA Rugosurf 90G (TESA technology, Swizerland) under ISO 4287. Further, a MicroXAM-800 (KLA-Tencor, USA) 3D noncontact surface-mapping profiler was used to investigate the topography of the wear tracks.

\subsubsection{Microscopic finite-element analysis}

To further analyse the mechanical behaviour and stability of NT and SO-NT in detail, microscopic finite-element (FE) models were established with the ANSYS software. Unit bending and twisting loads were applied to the FE models of both NT and SO-NT, respectively. The deformations and stresses, which represent the material stiffness and strength, were obtained by conducting a statistical analysis.

\subsubsection{Corrosion behaviour}

Since NT could be applied to implant surfaces that are commonly surrounded by blood-rich tissue, the corrosion resistance of SO-NT should be evaluated. The open-circuit potentials (OCPs) of the NT and SO-NT samples were measured in a simulated body fluid (SBF). The electrochemistry measurements were carried out with the electrochemical workstation CHI660D (CH Instruments, Inc, Shanghai, China) to determine the corrosion resistance of SO-NT and NT by measuring the OCP versus time in SBF. The apparatus for the electrochemical measurements consisted of a three-electrodes electrotank with an OCP of $\pm 250 \mathrm{mV}$ and a scan rate of $0.3 \mathrm{mV} \mathrm{s}^{-1}$ at $22 \pm 1{ }^{\circ} \mathrm{C}$, a saturated calomel electrode for reference, and a platinum foil as counter electrode. The polarisation was recorded after the sample was soaked into SBF for 60 min until stabilisation. The scan data was recorded for $1500 \mathrm{~s}$. 
The Tafel polarization curve of Ti, NT and SO-NT were also been measured by the electrochemical workstation CHI660D (CH Instruments, Inc, Shanghai, China) under room temperature. The bare Ti specimen was a control reference to comparative analyze the corrosion parameters of NT and SO-NT. The scan rate $(\mathrm{V} / \mathrm{s})$ was 0.01 , initial from $E(V)=-1$ to final $E(V)=0$.

\section{Results and Discussion}

\subsection{Physicochemical Characterisation}

\subsubsection{Nanostructure analysis}

The top views of NT and SO-NT showing porous structures with inner diameters of $100 \mathrm{~nm}$ were similar. Analyses of the cross-sections of SO-NT and NT are shown in Figure $2 \mathrm{a}$ and $2 \mathrm{~b}$. A $120-150 \mathrm{~nm}$ thick interfacial $\mathrm{TiO}_{2}$ bonding layer was formed at the NT-Ti interface. The interfacial layer was wrapped around the NT bottoms with no clear boundary between the interface of tube bottom and layer (Figure 2a). Owing to the increased contact area of the tube bottoms, the interfacial layer compensates shear forces and bore loadings. In the bottom view of the NT and SO-NT (Figure $2 \mathrm{c}$ and 2d), it can be noted that the SO-NT bottom became flat with tiny bulges, which were distributed at the bottom outer-shells of the half-spherical NT. Regarding the NT sample, a typical tube bottom structure with hexagonal half-spheres and clear edges can be observed in each NT contact area.

\subsubsection{XPS analysis}

The chemical compositions of the NT and SO-NT samples were measured via XPS with a survey scan and high-resolution element spectra for phosphorus and fluorine (Figure 3). The XPS full-spectrum and high-resolution scan of SO-NT indicates a phosphorous peak (P2p). Studies on phosphorous-incorporated Ti surfaces have demonstrated an enhanced osteoblast attachment, os- 
teoblast gene expression, and removal torque forces [30]. According to studies, the intensity of fluorine (F1s) was significantly lower after an additional anodisation. Further, it is also established that a fluorosis can increase the bone fracture risk [31, 32]. The here presented SO-NT sample exhibited an extreme rare fluoride concentration and displayed existence of phosphorous: an evident existence of phosphorous in an elemental composition similar to the mineral phase of natural bone tissue was observed [33]. As a result of surface modification, the surface wettability of both NT and SO-NT surfaces have been improved, as reported in Figure S2. The elements distribution by EDS mapping measurement of NT and SO-NT were shown in Figure S3. It was shown that titanium, oxygen and fluorine were distributed homogenously within the top layer on NT surface. As for element distribution on SO-NT surface, it was also observed that, in addition to element titanium, oxygen, fluorine which were uniformly distributed uniformly, the phosphorus was introduced into the top layer of SO-NT specimen, as observed in Figure S3.

\subsection{Biotribology and Biocorrosion}

\subsubsection{Mechanical-stability evaluation}

Since a clinical insertion of an implant can cause micro-deformations in the implant, the coating material must be firmly attached to the implant. For comparison purposes, one half of the Ti plate was treated with a traditional oxidation to obtain common NT, whereas the rest was treated with a multi-step oxidation to obtain SO-NT. The differently coloured regions can be clearly distinguished in Figure 4a1. Owing to mechanical bending, one side of the Ti foil experienced strong tension (Figure 4a2), whereas the other side experienced strong compression (Figure 4a4). Regarding the side under tension, the NT layer was approximately completely peeled off of the Ti substrate, whereas no obvious damages in the SO-NT part could be observed (Figure 4a1). Regarding the side under compres- 
sion, the NT part exhibited a peeled debris, whereas no damages were found in the SO-NT part (Figure 4a3). Furthermore, a twisting test was performed to evaluate the adhesion strength of the NT layer on the Ti substrate. After several twists (Figure 4b2), the NT layer of the NT part completely peeled off, whereas the SO-NT layer remained intact (Figure 4b3, Movie 1). Next, a impact test was conducted to prove the improved mechanical stability of SO-NT (Figure 4c2): The NT layer of the NT part approximately completely peeled off of the substrate, whereas the NT layer of the SO-NT part remained adhered to the substrate (Figure 4c3, Movie 2). Further, HR-SEM was employed to investigate the surface morphology after hitting. As shown in Figures 4 c3-1-c3-3, no NT layer was left in the NT-Ti part, whereas it remained intact in the SO-NT part but was covered by many fragments, and the NT topography could still be detected in SO-NT areas. Subsequently, the falling-ball impact experiment was performed on the NT and SO-NT samples, respectively. Heavy damages on the NT sample due to stripped NT could be observed (Figure 4d2). By contrast, approximately no damages were observed on the SO-NT surface, indicating a strong bonding between NT layer and Ti substrate (Figure 4d3). A mechanical ultrasonic measurement was carried out to evaluate the mechanical stability of both samples (Figure S4). The NT layer tended to peel off of the Ti substrate with increasing ultrasonic exposure time and was approximately completely removed after $180 \mathrm{~s}$. By contrast, no damages in the NT layer on the Ti substrate of the SO-NT sample were found. The aforementioned results indicate that the as-prepared SO-NT sample possessed a strong mechanical stability compared with the traditional NT sample. Such excellent mechanical properties enhance the reliability of SO-NT with respect to their use in medical implants in the field of tissue engineering.

Friction and wear tests were conducted to quantitatively investigate the mechanical property under dynamic shearing. A ball-on-disk contact mode was used to investigate the tribological prop- 
erty. As shown in Figure S5, no significant differences in the coefficient of friction (COF) were found for the SO-NT and NT samples after 7000 sliding cycles under approximately 12 MPa of Hertzian contact pressure. The COFs of NT and SO-NT were both $0.2( \pm 0.02)$. However, obvious signal fluctuations in the friction curve and the stick-slip phenomenon were observed for NT until 5000 cycles. Thereafter, its COF slightly decreased, thereby suggesting a minorly damaged NT structure of the NT sample.

After the friction tests, the morphologies of the wear tracks were investigated. As shown in Figure 5 a1 and b1, the widths of the wear tracks of the NT sample were approximately two times those of the SO-NT sample. The centre area of the wear tracks was selected to characterise the micro-area topography of scratches using a 3D MicroXAM-800 (Figure 5 a2 and b2). Micro-sized ploughs were distributed on both samples, whereas the SO-NT sample exhibited less ploughs with clearer edges (Figure 5b2). The ploughs were further investigated via HR-SEM. Examples can be seen in Figure 5 a3 and b3. The plough width on the NT sample was $25 \pm 1 \mu \mathrm{m}$, which was approximately two times the width of that on the SO-NT sample $(10 \pm 1 \mu \mathrm{m})$. Further, mass cracks in the vicinity of the plough on the NT surface were observed. By contrast, the SO-NT surface exhibited no cracks.

A six-stations pin-on-disk procedure was conducted to quantify the volume loss of the NT and SO-NT samples after the wear tests (Figure S1). After 12000 test cycles, the wear morphologies and wear volumes were examined. As shown in Figure 6a, the surfaces of three NT samples exhibited heavy wear and the Ti substrates were exposed, whereas light wear tracks were observed on the SO-NT samples. In addition, the morphologies of the spherical-ended stainless steel pins were examined. The wear tracks of the pins encountering NT were heavy and those encountering SO-NT were light. To quantitatively analyse the volume loss of wear tracks for disks and pins, the theoretical 
model in [34] can be employed. The calculation results showed that the volume losses were 0.48 $\mathrm{mm}^{3}$ and $0.032 \mathrm{~mm}^{3}$ for the NT disks and pins encountering the NT, respectively. By contrast, the volume losses were only $0.07 \mathrm{~mm}^{3}$ and $0.007 \mathrm{~mm}^{3}$ for the SO-NT disks and pins, respectively. The excellent anti-wear property of the SO-NT samples can be attributed to the strong adherence of the bonding layer to the two interfaces (NT-bonding layer and bonding layer-Ti) rather than to the changing mechanical modulus of the NT layer grown on the NT and SO-NT samples, which can be proved via nanoindentation (Figure S6).

\subsubsection{Microscopic finite-element analysis and mechanism discussion}

As shown in Figure 7a, the maximal deformations of NT and SO-NT under unit bending loads were $0.029 \mu \mathrm{m}$ and $0.009 \mu \mathrm{m}$, respectively. The maximal equivalent stresses were $108 \mathrm{MPa}$ and 63 MPa, respectively. Compared with NT, the maximal deformation of SO-NT was decreased by $68.97 \%$ and the maximal equivalent stress was decreased by $41.67 \%$.

The maximal deformations of NT and SO-NT under unit twisting loads were $0.087 \mu \mathrm{m}$ and 0.025 $\mu \mathrm{m}$, respectively; The maximal equivalent stresses were $230 \mathrm{MPa}$ and $72 \mathrm{MPa}$, respectively (Figure 7b). Compared with NT, the maximal deformation of SO-NT was decreased by $71.26 \%$ and the maximal equivalent stress was decreased by $68.70 \%$. The results also show that the stress concentration of SO-NT was evidently reduced. Hence, the stiffness and strength were enhanced owing to the existence of the bonding layer.

According to the experimental results and FE analysis, the produced SO-NT samples exhibited excellent mechanical stabilities under harsh bending, twisting, impact, ultrasonic radiation, and wear. This results from the special stress dissipation in SO-NT under shearing. Regarding the traditional NT samples, the accumulation of external stress caused an irregular arrangement of the once 
vertically arranged NT. The aggregation of disordered NT generated cracks, which led to the peel-off of the NT layer from the Ti substrate (Figure 8a). By contrast, SO-NT exhibited an impressive dissipation with no crack generation in the vicinity of the wear track. This observation indicates the possibly advantageous mechanisms of the SO-NT sample compared to conventional NT samples (Figure 8b). Furthermore, the thickness plays a dominate role by affecting the adhesion strength; $\mathrm{A}$ thicker anodic layer tends to detach [8]. However, the bonding layer in this study measured only 120-150 nm. Apart from these two characteristics, the improved mechanical stability of SO-NT is possibly related to the strong bonding at the two interfaces (bonding layer-Ti substrate and bonding layer-NT layer). The strong bonding interfaces are essential for a continuous dissipation of external stress without stress concentrations at the half-spherical bottoms of the NT layer. The HR-SEM bottom view (Figure 2c) shows that the contact area of the SO-NT-Ti interface was covered by the bonding layer. With bonding layer, the original NT bottom with half-spheres exhibited tiny bulges and the bonding layer filled the space between NT bottoms and Ti substrate, which was generated by fluorine ions during anodisation. According to the cross-section images (Figure 2a), no edge existed at the bonding layer-NT interface. Hence, the bonding layer was tightly wrapped around the NT bottoms. Another possible mechanism is the altered stress dissipation of the NT layer through the bonding layer. Owing to the strong bonding at the bonding layer-NT interface, the stress is distributed and dissipated. The subsistence of a hyperfine bonding layer with $100 \mathrm{~nm}$ grain (Figure S7) contributes to a reduction of stress concentrations via a stress transmission from the in-situ tube bottom to grains on the bonding layer and adjacent tubes, thereby acting as a ground foundation. The assumption is confirmed by the analysis on wear ploughs and wear tracks (Figure 5 a3 and 4b3; Figure 6a).

\subsubsection{Corrosion behaviour}


After the samples were soaked for $60 \mathrm{~min}$ in SBF, the OCP was recorded for $1500 \mathrm{~s}$. As shown in

Figure 9, SO-NT (approximately -0.06 V) presented a lower corrosion tendency than NT (approximately $-0.28 \mathrm{~V})$. The OCP value indicates the sample surface activation, which can be active or passive. The electrochemical attack in SBF, which can erode the sample surface, leads to an ion release from the substrate into the medium. The Tafel polarization curve has also demonstrated the improved corrosion resistance of SO-NT than NT and bare Ti (Figure S8). Apart from corrosion potential, corrosion current density is another significant parameter which is proportional to current rate. The corrosion current density of bare Ti and NT were measured as $0.77 \mu \mathrm{A} / \mathrm{cm}^{2}$ and $14.41 \mu \mathrm{A} / \mathrm{cm}^{2}$, whereas the value of SO-NT was $0.07 \mu \mathrm{A} / \mathrm{cm}^{2}$. Obviously, SO-NT has significantly reduced the corrosion potential and corrosion current density.

The erosion process of NT can be initiated in locations where the fluid directly touches the Ti substrate through slots or structural defects between adjacent tubes, thereby attacking the Ti-NT bottom interface. The ions from the adjacent substrate can be transmitted to the area of initiated erosion and react with the ions in the media, which leads to point corrosions at the NT-Ti interface. Further, the process might increase the ion release of $\mathrm{Ti}$ and generate micro pits at the interface, which would cause a lift-off of the NT layer in the long term. In this study, the 120-150 nm dense bonding layer acted as barrier to isolate the SBF solution from the slots between NT (Figure 10). Moreover, the barrier was tightly connected to the NT bottoms (Figure 2a) and prevented the media from reaching the Ti substrate. This characteristic promotes a passive electrochemical environment and improves the SO-NT corrosion resistance.

\section{Conclusion}


To improve the mechanical stability of materials with traditional $\mathrm{TiO}_{2}$ nanotubes (NT)-in particular, their biotribological performance-for potential applications as implants, we prepared structure-optimised $\mathrm{TiO}_{2}$ nanotubes (SO-NT) by creating an interfacial bonding layer at an NT layer-titanium (Ti) substrate interface. The HR-SEM results showed a 120-150 $\mathrm{nm}$ bonding layer, which was completely wrapped around the bottoms of the NT array and tightly attached to the Ti substrate. Compared with the manufactured conventional NT material, the manufactured SO-NT material showed an enhanced mechanical stability, which was confirmed via several reliable experimental evaluations including bending, twisting, impact, and ultrasonic-radiation tests. Furthermore, according to the biotribological tests, the SO-NT exhibited an improved anti-wear behaviour. The bonding layer could effectively resist a peel-off and improve the mechanical stability of the NT array under dynamic shearing. Moreover, electrochemical tests revealed that the produced SO-NT material possessed an improved anti-corrosion behaviour according to its higher open-circuit potential (-0.06 V) compared to that of NT $(-0.26 \mathrm{~V})$ in SBF. The potential responsible mechanism is the barrier-like behaviour of the bonding layer, which prevents the Ti substrate from eroding. In conclusion, owing to its improved stability concerning the nanostructured surface, the SO-NT demonstrates a great potential compared to conventional NT for implant applications, such as orthopaedic and dental implants as well as implants interfacing bone tissue.

\section{Acknowledgments}

This project is financially supported by the EU via the H2020-MSCA-RISE-2016 program (grant no: 734156), Royal Society via the International Exchange Program (grant no: IE161349), Key Research Project from the National Key Research and Development Program of China (2016YFC1100401), Na- 
tional Natural Science Foundation of China (51705507), and Young Elite Scientists Sponsorship Program by CAST (2017QNRC001).

\section{References}

[1] Lee K, Mazare A, Schmuki P. One-dimensional titanium dioxide nanomaterials: nanotubes. Chemical Reviews, 2014, 19, 9385-9454.

[2] Macak J M, Tsuchiya H, Taveira L, Aldabergerova S, Schmuki P. Smooth anodic TiO2 nanotubes. Angewandte Chemie International Edition, 2005, 45, 7463-7465.

[3] Brammer K S, Frandsen C J, Jin S. TiO2 nanotubes for bone regeneration. Trends Biotechnology, 2012, 6, 315-322.

[4] Wang F, Shi L, He W X, Han D, Yan Y, Niu Z Y, Shi S G. Bioinspired micro/nano fabrication on dental implant-bone interface, Applied Surface Science, 2013, 265, 480-488.

[5] Rho J Y, Kuhn-Spearing L, Zioupos P. Mechanical properties and the hierarchical structure of bone, Medical engineering \& physics, 1998, 20, 92-102.

[6] Zhang L J, Webster T J. Nanotechnology and nanomaterials: Promises for improved tissue regeneration, Nano Today, 2009, 4, 66-80.

[7] Mendonca G, Mendonca D B, Aragao F J, Cooper L F. Advancing dental implant surface technology-From micron-to nanotopography, Biomaterials, 2008, 29, 3822-3835.

[8] Li Y, Lee I S, Cui F Z, Choi S H. The biocompatibility of nanostructured calcium phosphate coated on micro-arc oxidized titanium, Biomaterials, 2008, 29, 2025-2032.

[9] Palin E, Liu H, Webster T J. Mimicking the nanofeatures of bone increases bone-forming cell adhesion and proliferation, Nanotechnology, 2005, 16, 1828-1835.

[10] Wang N, Li H Y, Lu W L, Li J H, Wang J S, Zhang Z T, Liu Y R. Effects of TiO2 nanotubes with different diameters on gene expression and osseointegration of implants in minipigs. Biomaterials, 2011, 29, 6900-6911.

[11] Kwon D H, Lee S J, Wikesjo U M E, Johansson P H, Johansson C B, Sul Y T. Bone tissue response following local drug delivery of bisphosphonate through titanium oxide nanotube implants in a rabbit model. Journal of Clinical Periodontology, 2017, 9, 941-949. 
[12] Shen X K, Ma P P, Hu Y, Xu G Q, Xu K, Chen W Z, Ran Q C, Dai L L, Yu Y L, Mu C Y, Cai K Y. Alendronate-loaded hydroxyapatite-TiO2 nanotubes for improved bone formation in osteoporotic rabbits. Journal of Materials Chemistry B, 2016, 8, 1423-1436.

[13] Salou L, Hoornaert A, Louarn G, Layrolle P. Enhanced osseointegration of titanium implants with nanostructured surfaces: an experimental study in rabbits. Acta Biomaterialia, 2015, 11, 494-502.

[14] Yi Y A, Park Y B, Choi H, Lee K W, Kim S J, Kim K M, Oh S, Shim J S. The evaluation of osseointegration of dental implant surface with different size of $\mathrm{TiO} 2$ nanotube in rats. Journal of Nanomaterials, 2015, 2, 1-11.

[15] von Wilmowsky C, Bauer S, Lutz R, Meisel M, Neukam F W, Toyoshima T, Schmuki P, Nkenke E, Schlegel K A. In vivo evaluation of anodic TiO2 nanotubes: an experimental study in the pig. Journal of Biomedical Materials Research Part B: Applied Biomaterials, 2009, 1, 165-171.

[16] Geetha M, Singh A K, Asokamani R, Gogia A K. Ti based biomaterials, the ultimate choice for orthopaedic implants-a review. Progress in materials science, 2009, 3, 397-425.

[17] Raphel J, Holodniy M, Goodman S B, Heilshorn S C. Multifunctional coatings to simultaneously promote osseointegration and prevent infection of orthopaedic implants. Biomaterials, 2016, 84, 301-314.

[18] Cvijović-Alagić I, Cvijović Z, Mitrović S, Panić V, Rakin M. Wear and corrosion behavior of Ti-13Nb-13Zr and Ti-6Al-4V alloys in simulated physiological solution. Corrosion Science, 2011, 2, 796-808.

[19] Hacisalihoglu I, Samancioglu A, Yildiz F, Purcek G, Alsaran A. Tribocorrosion properties of different type titanium alloys in simulated body fluid. Wear, 2015, 332, 679-686.

[20] Ma M H, Kazemzadeh-Narbat M, Hui Y, Lu S S, Ding C F, Chen D D Y, Hancock R E W, Wang R Z. Local delivery of antimicrobial peptides using self-organized $\mathrm{TiO} 2$ nanotube arrays for peri-implant infections. Journal of Biomedical Materials Research Part A, 2012, 100(2), 278-285.

[21] Oh S, Brammer K S, Li Y J, Teng D, Engler A J, Chien S, Jin S. Stem cell fate dictated solely by altered nanotube dimensions. Proceedings of the National Academy of Sciences, 2009, 106(7), 2130-2135.

[22] Qiao H X, Xiao H Y, Huang Y, Yuan C F, Zhang X J, Bu X P, Wang Z H, Han S G, Zhang L J, Su Z B, Zhang X J. SiO2 loading into polydopamine-functionalized $\mathrm{TiO} 2$ nanotubes for biomedical applications. Surface and Coatings Technology, 2019, 364: 170-179. 
[23] Huang Y, Shen X, Qiao H X, Yang H, Zhang X J, Liu Y Y, Yang H J. Biofunctional Sr-and Si-loaded titania nanotube coating of Ti surfaces by anodization-hydrothermal process. International journal of nanomedicine, 2018, 13: 633-640.

[24] Xiong J Y, Wang X J, Li Y C, Hodgson P D. Interfacial chemistry and adhesion between titanium dioxide nanotube layers and titanium substrates. The Journal of Physical Chemistry C, 2011, 115(11), 4768-4772.

[25] Ouyang H M, Fei G T, Zhang Y, Su H, Jin Z, Xu S H, Zhang L D. Large scale free-standing open-ended TiO2 nanotube arrays: stress-induced self-detachment and in situ pore opening. Journal of Materials Chemistry C, 2013, 1(45), 7498-7506.

[26] Wang D A, Hu T C, Hu L T, Yu B, Xia Y Q, Zhou F, Liu W M. Microstructured Arrays of TiO2 Nanotubes for Improved Photo-Electrocatalysis and Mechanical Stability. Advanced Functional Materials, 2009, 19(12), 1930-1938.

[27] Yu D L, Zhu X F, Xu Z, Zhong X M, Gui Q F, Song Y, Zhang S Y, Chen X Y, Li D D. Facile method to enhance the adhesion of TiO2 nanotube arrays to Ti substrate. ACS Applied Materials \& Interfaces, 2014, 6(11), 8001-8005.

[28] Macak J M, Albu S P, Schmuki P. Towards ideal hexagonal self-ordering of TiO2 nanotubes. physica status solidi (RRL)-Rapid Research Letters, 2007, 1(5), 181-183.

[29] Alves S A, Rossi A L, Ribeiro A R, Toptan F, Pinto A M, Celis J P, Shokuhfar T, Rocha, L A. Tribo-electrochemical behavior of bio-functionalized $\mathrm{TiO} 2$ nanotubes in artificial saliva: Understanding of degradation mechanisms. Wear, 2017, 384, 28-42.

[30] Park J W, Kim Y J, Jang J H, Kwon T G, Bae Y C, Suh J Y. Effects of phosphoric acid treatment of titanium surfaces on surface properties, osteoblast response and removal of torque forces. Acta Biomaterialia, 2010, 6(4), 1661-1670.

[31] Cooper LF, Zhou Y S, Takebe J, Guo J L, Abron A, Holmen A, Ellingsen J E. Fluoride modification effects on osteoblast behavior and bone formation at $\mathrm{TiO} 2$ grit-blasted $\mathrm{cp}$ titanium endosseous implants. Biomaterials, 2006, 27(6), 926-936.

[32] Aaseth J, Shimshi M, Gabrilove J L, Birketvedt G S. Fluoride: a toxic or therapeutic agent in the treatment of osteoporosis? The Journal of Trace Elements in Experimental Medicine: The Official Publication of the International Society for Trace Element Research in Humans, 2004, 17(2), 83-92. 
[33] Le Guehennec L, Soueidan A, Layrolle P, Amouriq Y. Surface treatments of titanium dental implants for rapid osseointegration. Dental Materials, 2007, 23(7), 844-854.

[34] Sampaio M, Buciumeanu M, Henriques B, Silva F S, Souza J C M, Gomes J R. Tribocorrosion behavior of veneering biomedical PEEK to Ti6Al4V structures. Journal of the Mechanical Behavior of Biomedical Materials, 2016, 54, 123-130.

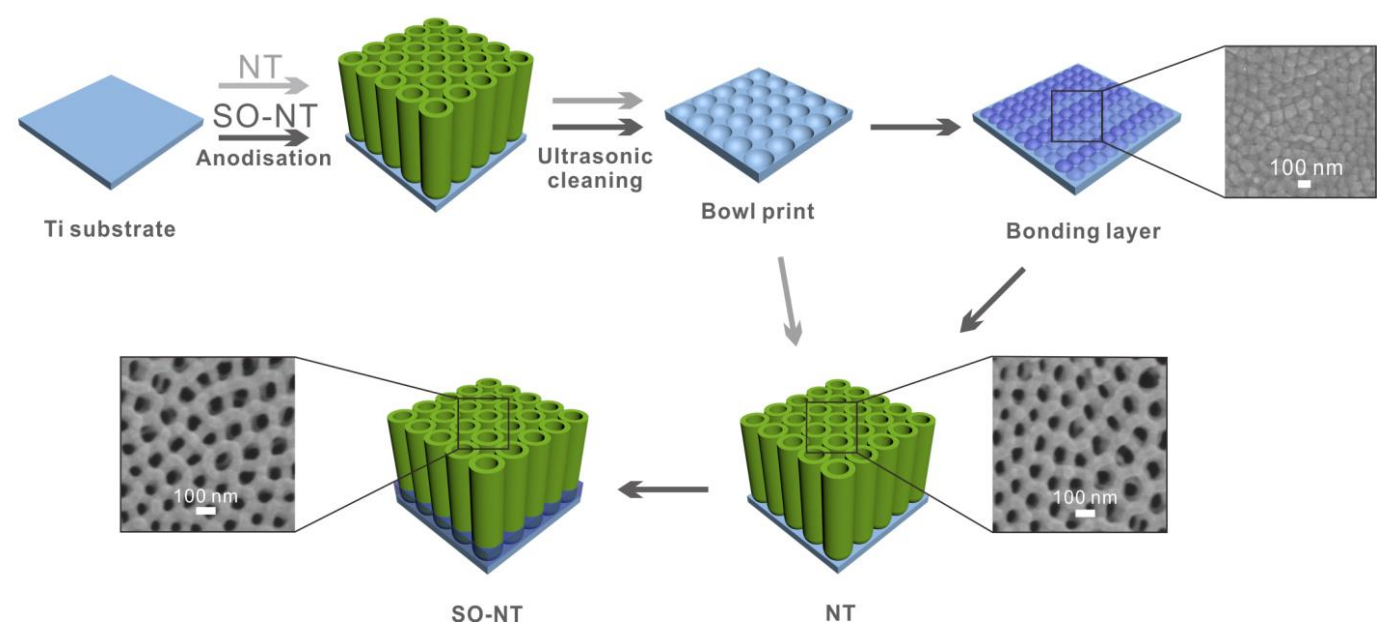

Figure 1. Fabrication of structure-optimised $\mathrm{TiO}_{2}$ nanotubes (SO-NT). 
1

2

3

5

6

7

8

9

10

11

12

13

14

15

16

17

18

19

20

21

22

23

24

25

26

27

28

29

30

31

32

33

34

35

36

37

38

39

40

41

42

43

44

45

46

47

48

49

50

51

52

53

54

55

56

57

58

59

60

61

62

63

64

65
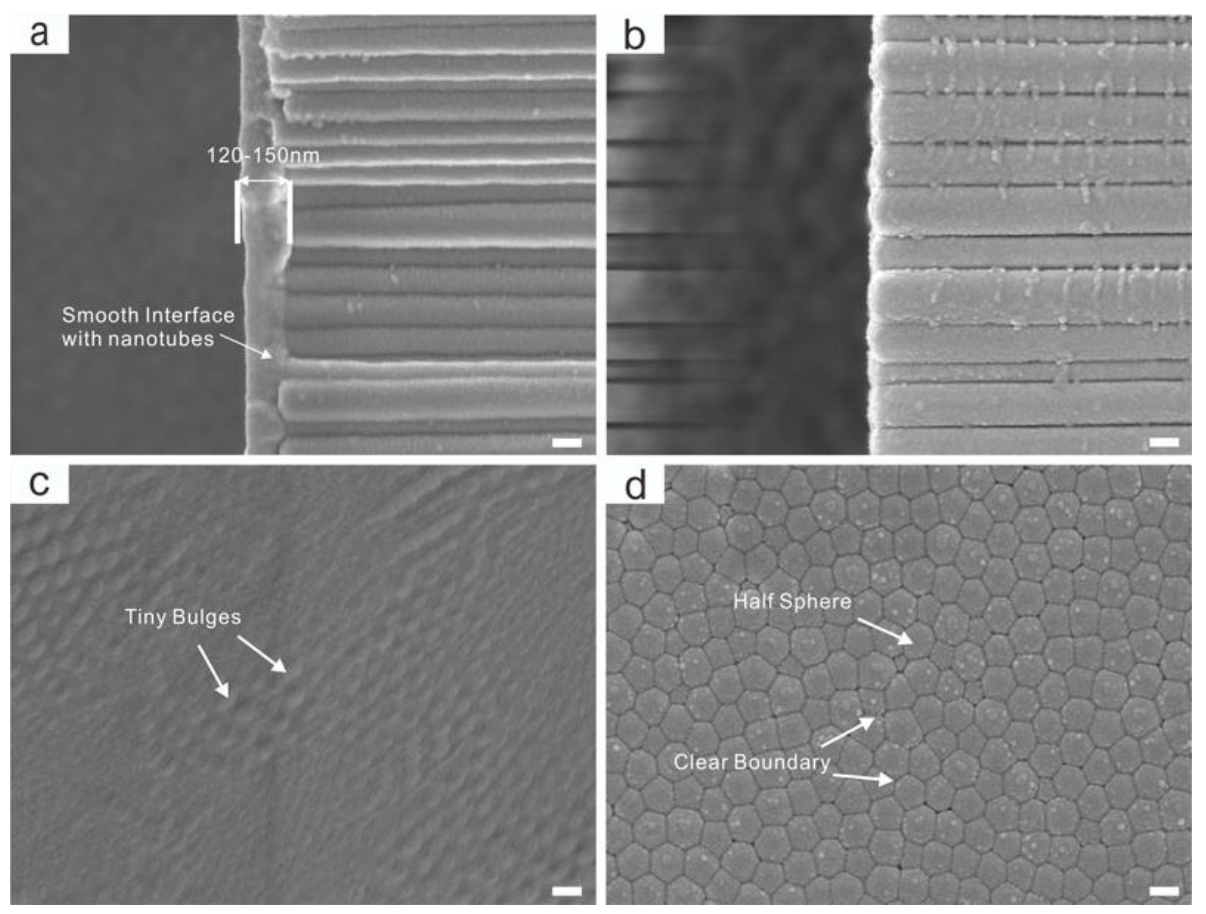

Figure 2. HR-SEM images of (a, c) SO-NT and (b, d) NT samples. The top and bottom images represent cross-sections and bottom morphologies (bar length $=100 \mathrm{~nm}$ ), respectively. 
1

2

3

4

5

6

7

8

10

11

12

13

14

15

16

17

18

19

20

21

22

23

24

25

26

27

28

29

30

31

32

33

34

35

36

37

38

39

40

41

42

43

44

45

46

47

48

49

50

51

52

53

54

55

56

57

58

59

60

61

62

63

64

65

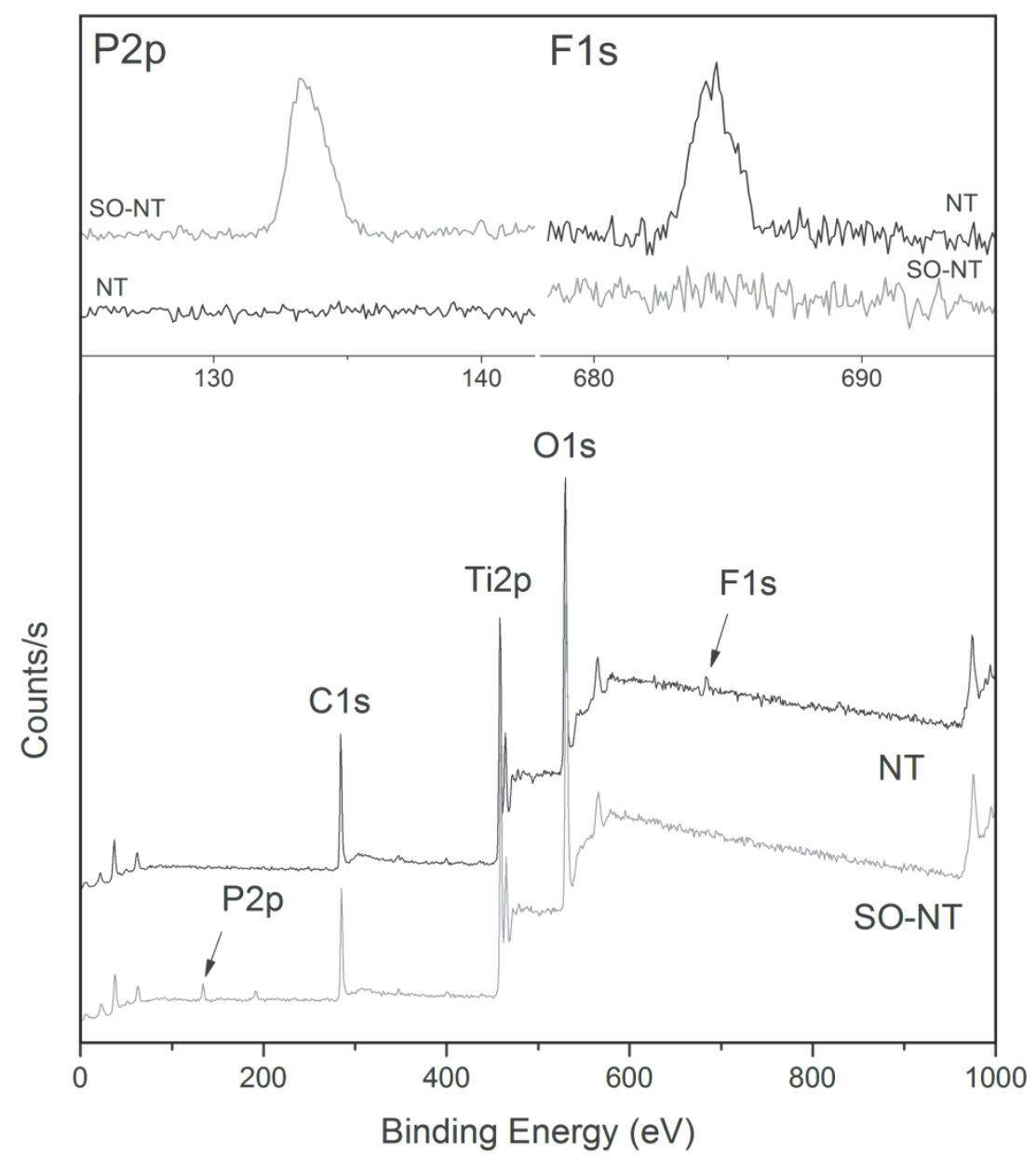

Figure 3. XPS spectra of NT and SO-NT samples and corresponding high-resolution scan peaks of F1s and P2p. 
1 a
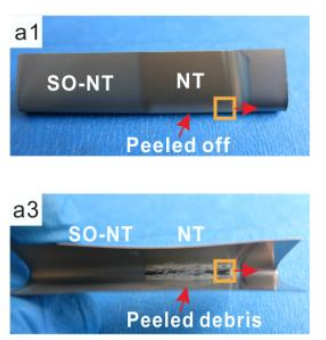

b

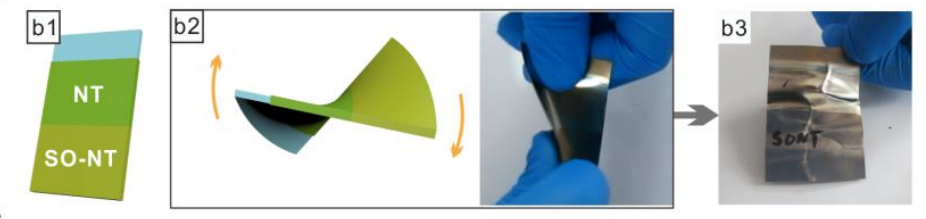

C
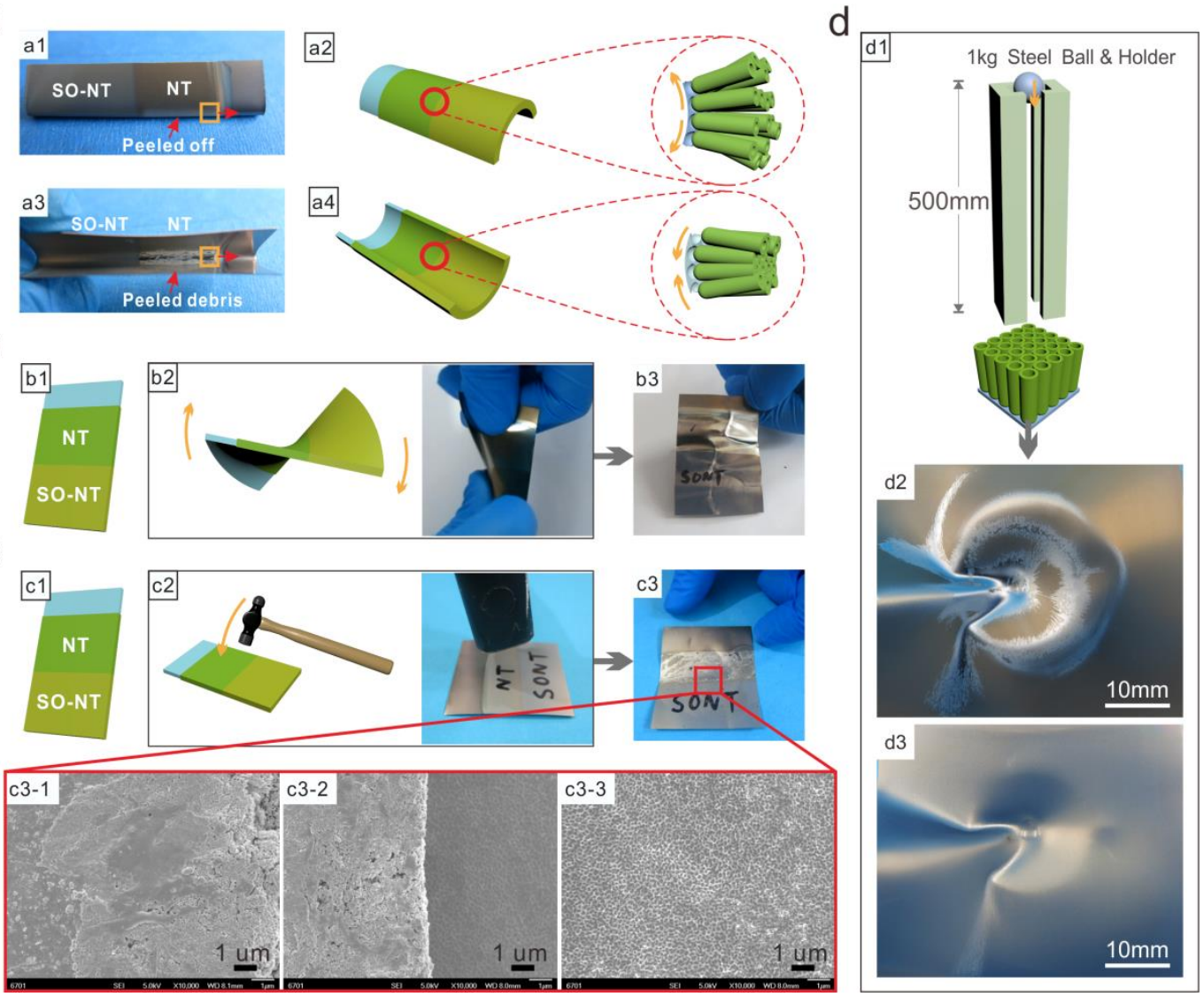

Figure 4. Evaluation of mechanical stability: (a) Bending, (b) twisting, and (c) impact tests on SO-NT and NT areas of one Ti sample. (d) Impact test: $1 \mathrm{~kg}$ steel holder with spherical tip was fixed at height of $500 \mathrm{~mm}$ to let the free-falling body shock the sample. 

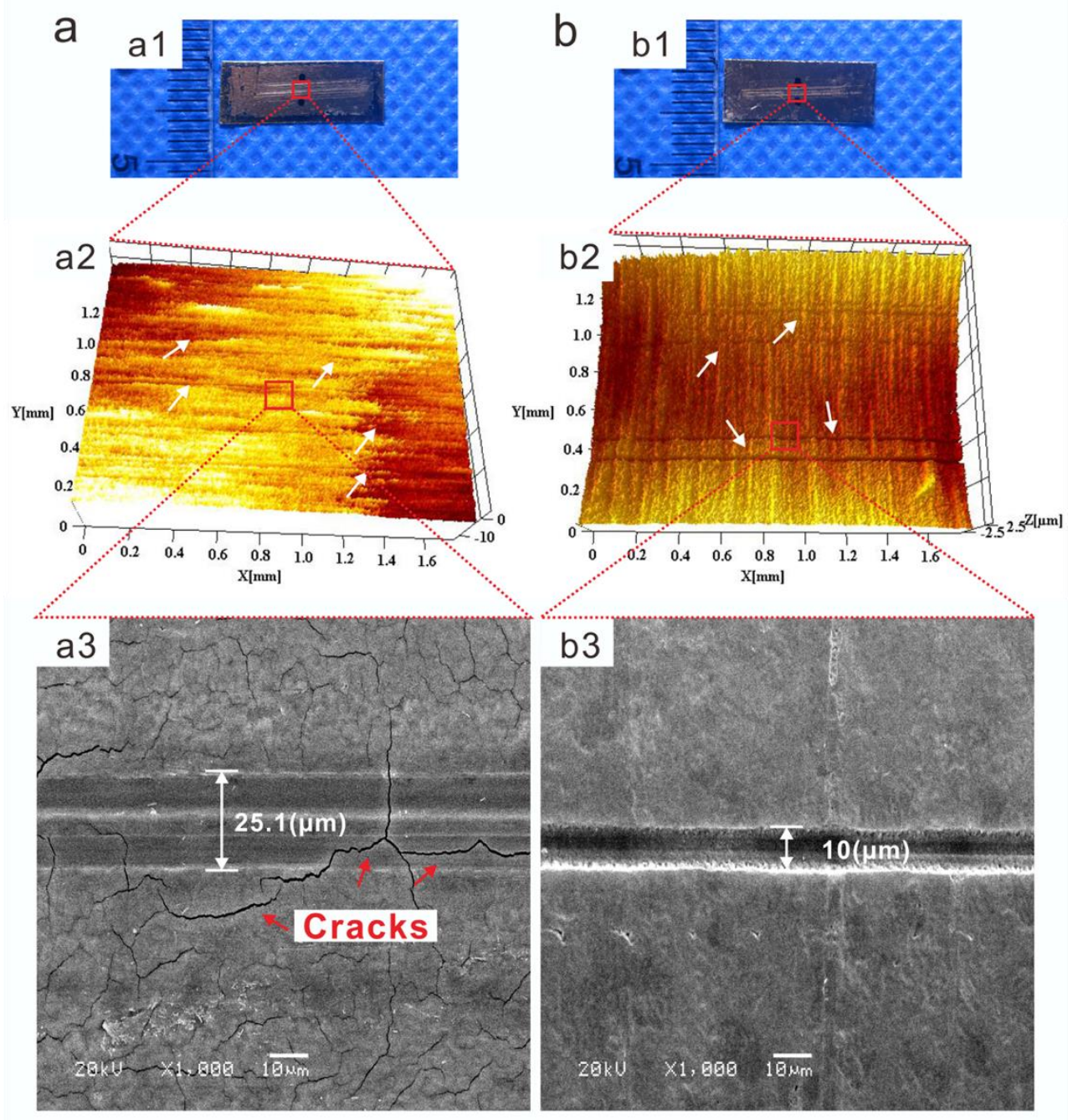

22

23

24

25

26

27

28

29

30

Figure 5. Wear track of (a) NT and (b) SO-NT samples. (a1,b1) Optical images, (a2,b2) topographs of centre area in wear tracks, $(\mathrm{a} 3, \mathrm{~b} 3)$ and SEM images of ploughs. 
1

2

3

5

6

7

8

9

10

11

12

13

14

15

16

17

18

19

20

21

22

23

24

25

26

27

28

29

30

31

32

33

34

35

36

37

38

39

40

41

42

43

44

45

46

47

48

49

50

51

52

53

54

55

56

57

58

59

60

61

62

63

64

65
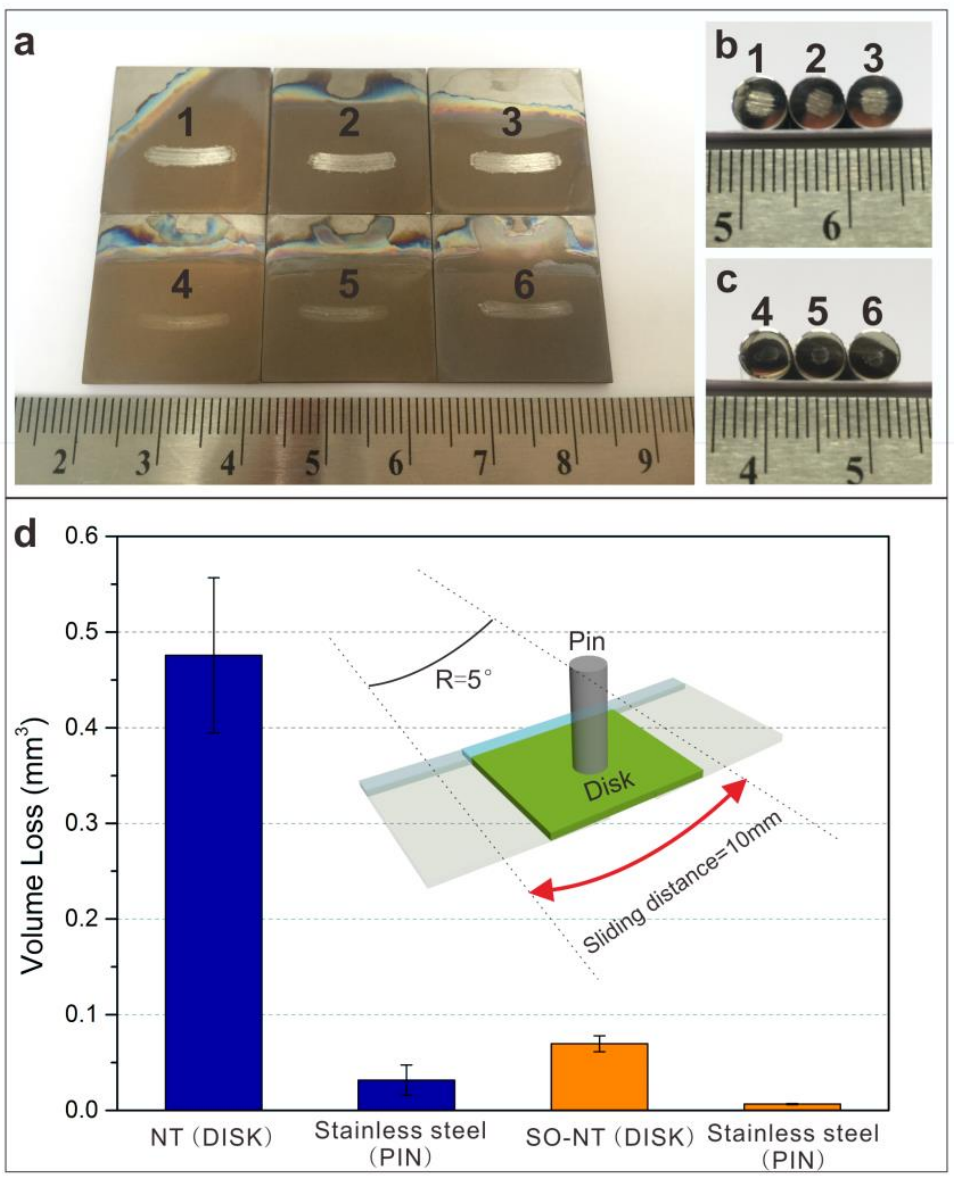

Figure 6. (a) Photographs of wear tracks on disks and pins after 12000 test cycles (1-3: NT samples; 4-6: SO-NT samples). (b) Volume loss of disks and pins. 
1

2

3

4

5

6

7

8

9

10

11

12

13

14

15

16

17

18

19

20

21

22

23

24

25

26

27

28

29

30

31

32

33

34

35

36

37

38

39

40

41

42

43

44

45

46

47

48

49

50

51

52

53

54

55

56

57

58

59

60

61

62

63

64

65

\section{a Deformation Stres}
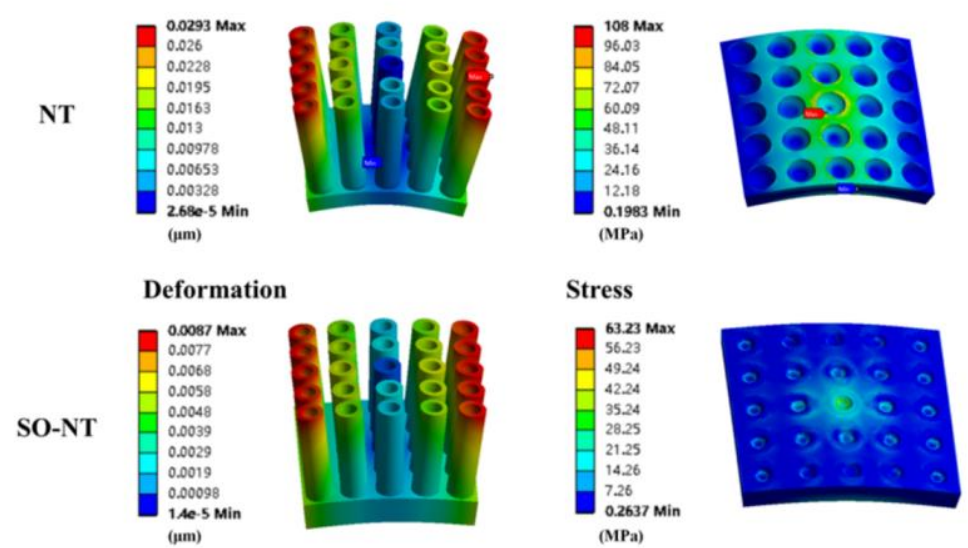

b Deformation

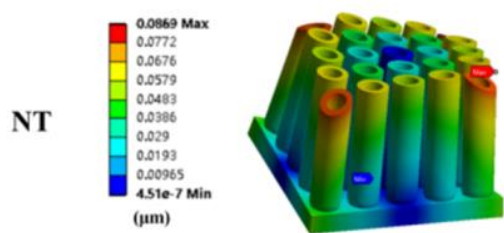

Stress
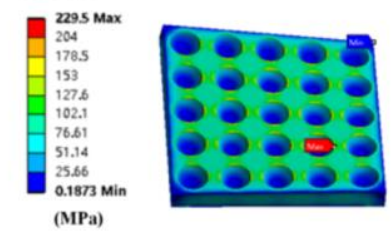

Stress

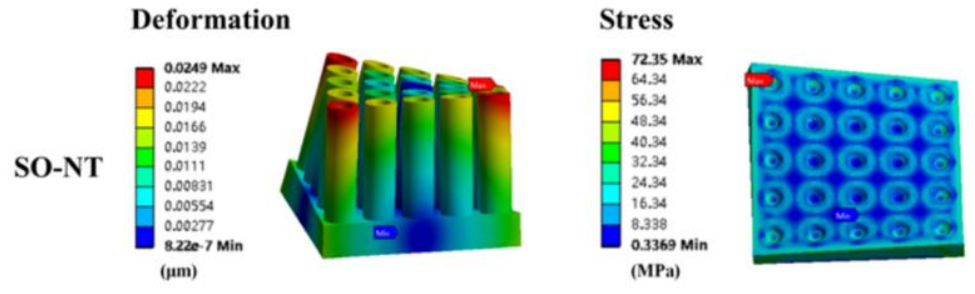

Figure 7. Deformation and stress results of FE simulations for (a) unit bending and (b) twisting load. 
1

2

3

4

5

6

7

8

9

10

11

12

13

14

15

16

17

18

19

20

21

22

23

24

25

26

27

28

29

30

31

32

33

34

35

36

37

38

39

40

41

42

43

44

45

46

47

48

49

50

51

52

53

54

55

56

57

58

59

60

61

62

63

64

65

a

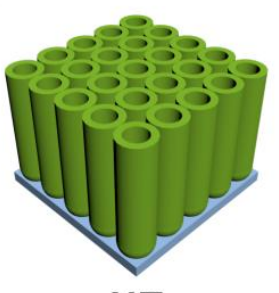

NT

b

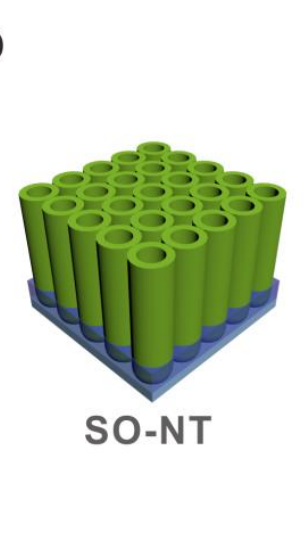

SO-NT
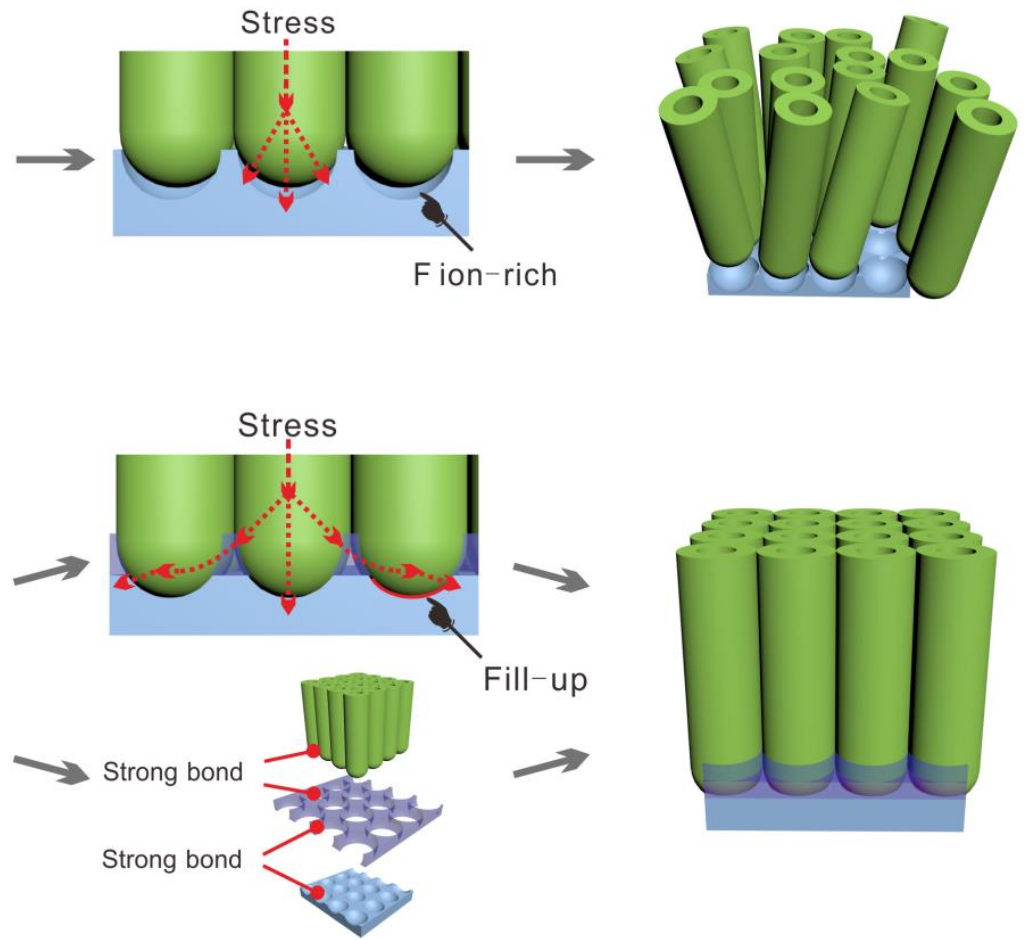

Figure 8. Mechanism responsible for mechanical stabilities of (a) NT and (b) SO-NT samples under stress. The red dotted arrow represents the direction of stress conduction.

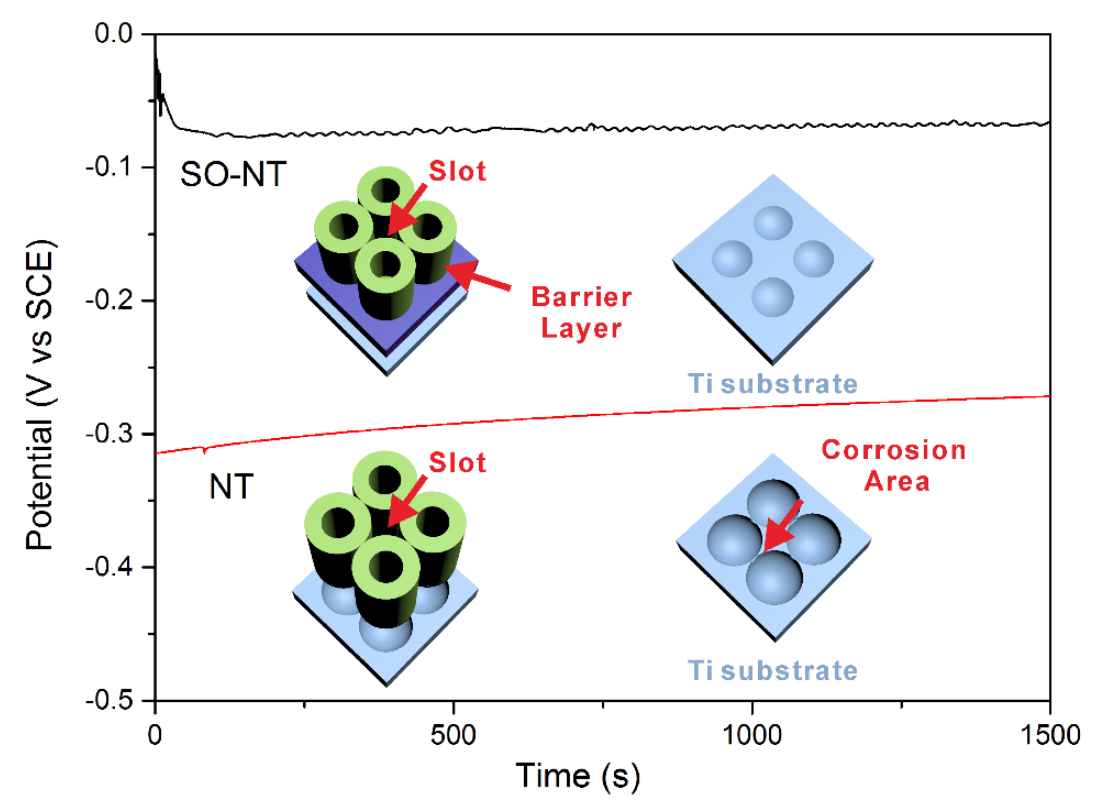

Figure 9. Open-circuit potential (OCP) versus time and 'barrier function' of interfacial bonding layer in SO-NT. 
1

2

3
4

5

6

7
8

8
9

10

11

12

13

14

15

16

17

18

19

20

21

22

23

24

25

26

27

28

29

30

31

32

33

34

35

36

37

38

39

40

41

42

43

44

45

46

47

48

49

50

51

52

53

54

55

56

57

58

59

60

61

62

63

64

65

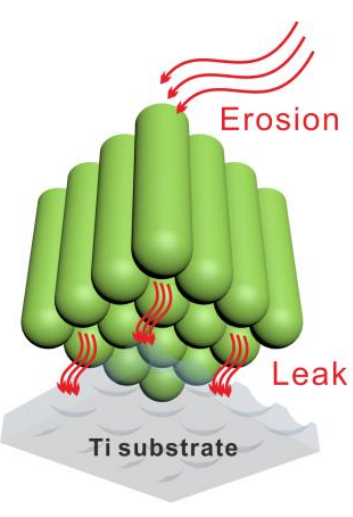

NT

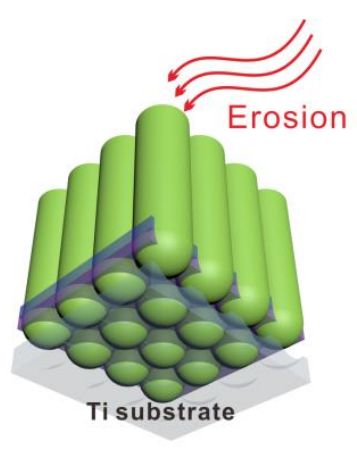

SO-NT

Figure 10. The bonding layer acts as isolating barrier. 


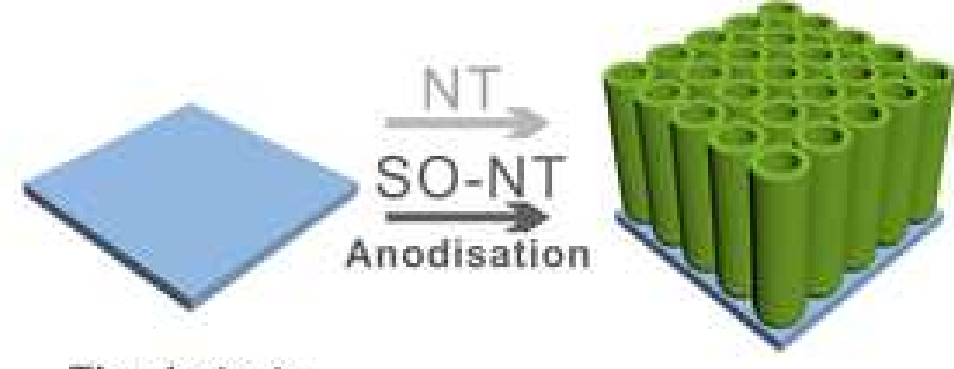

Ti substrate

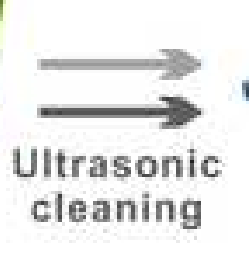

Uitrasonic Bowl print

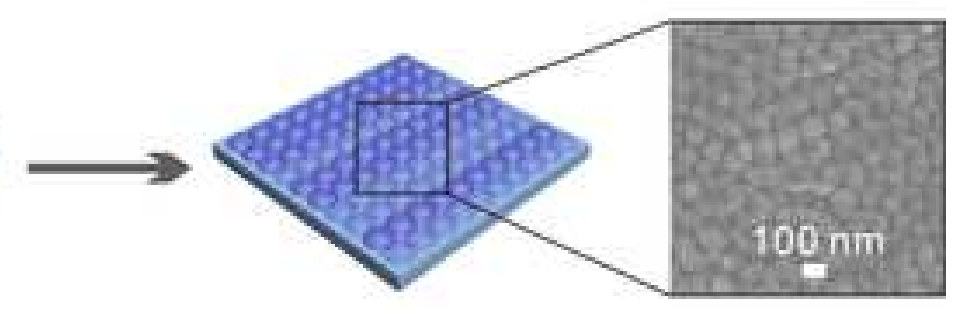

Bonding layer

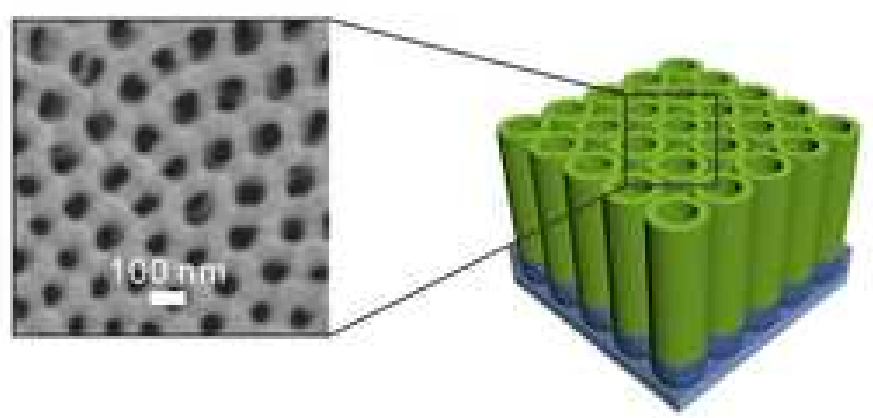

SO-NT
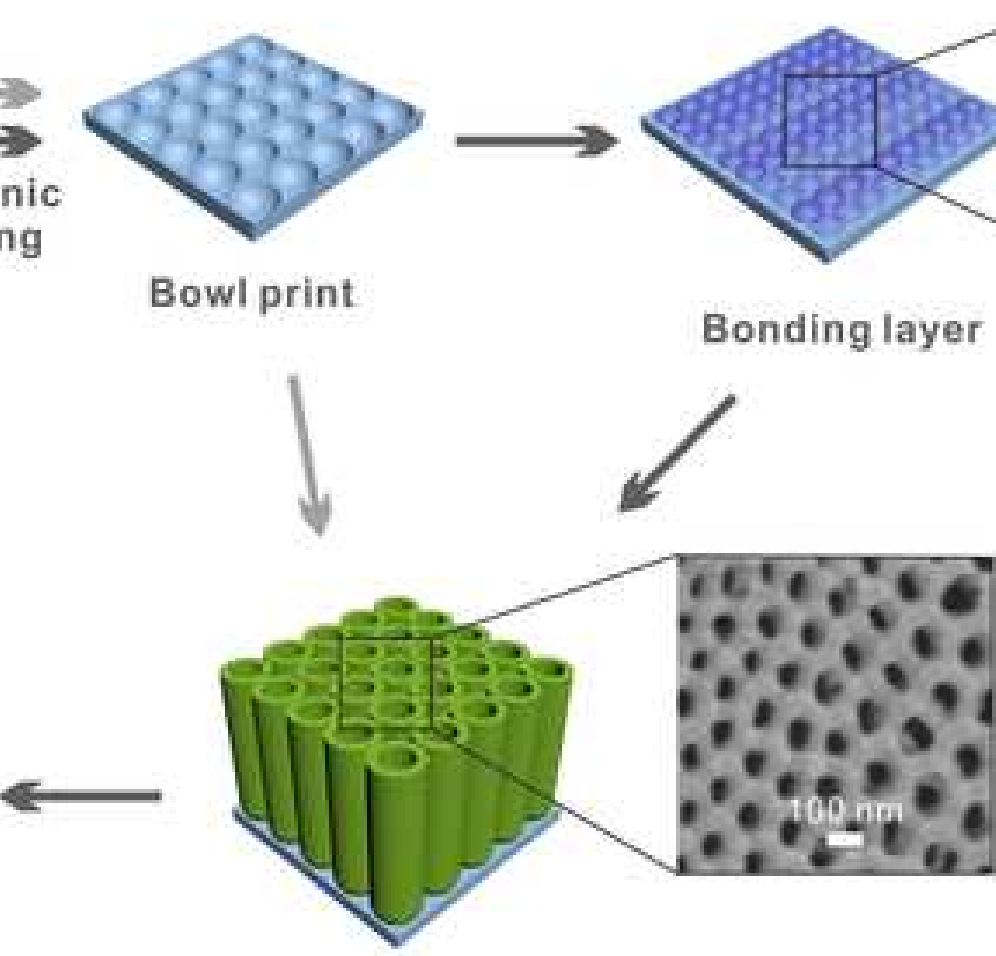

NT 


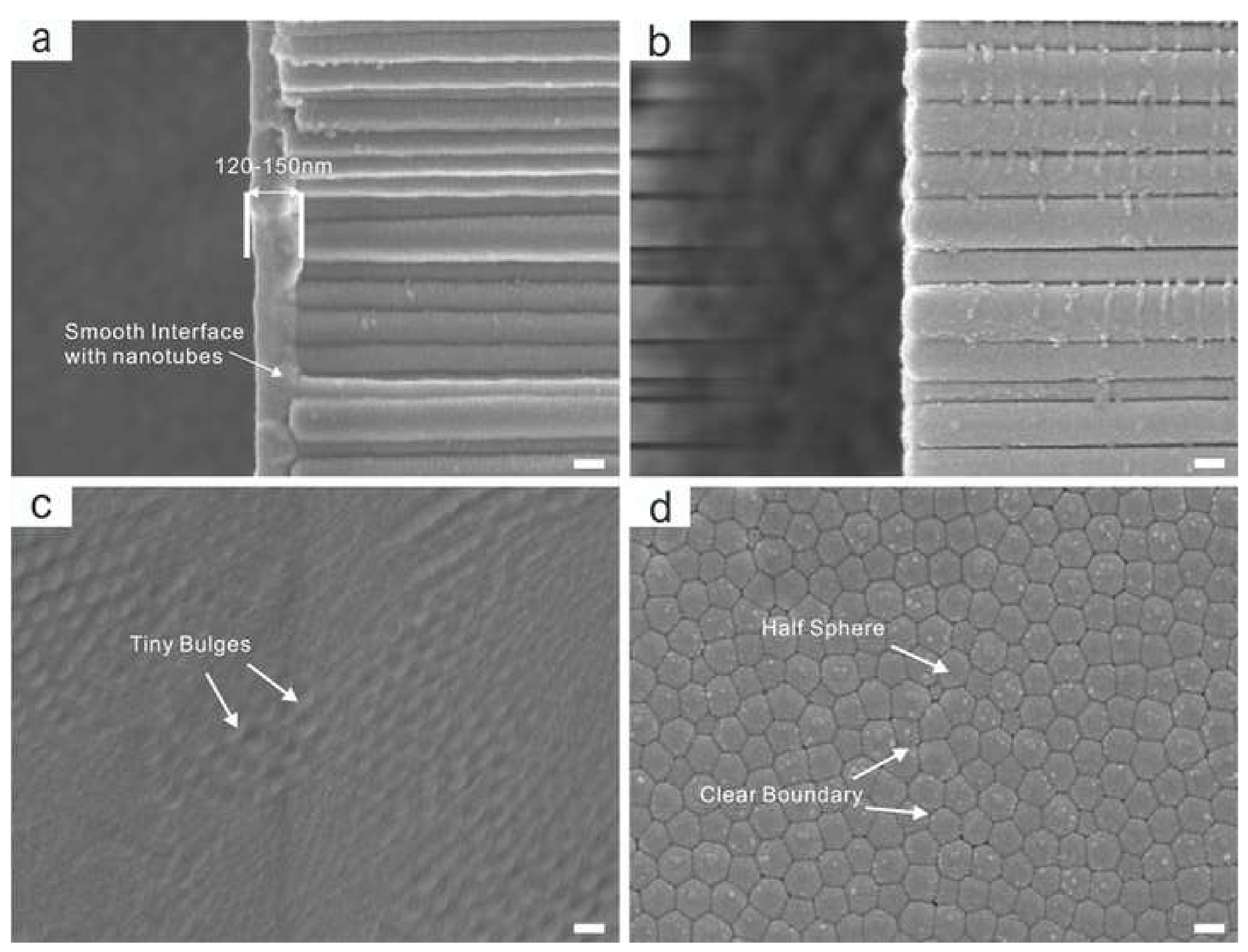

d

Clear Boundary 


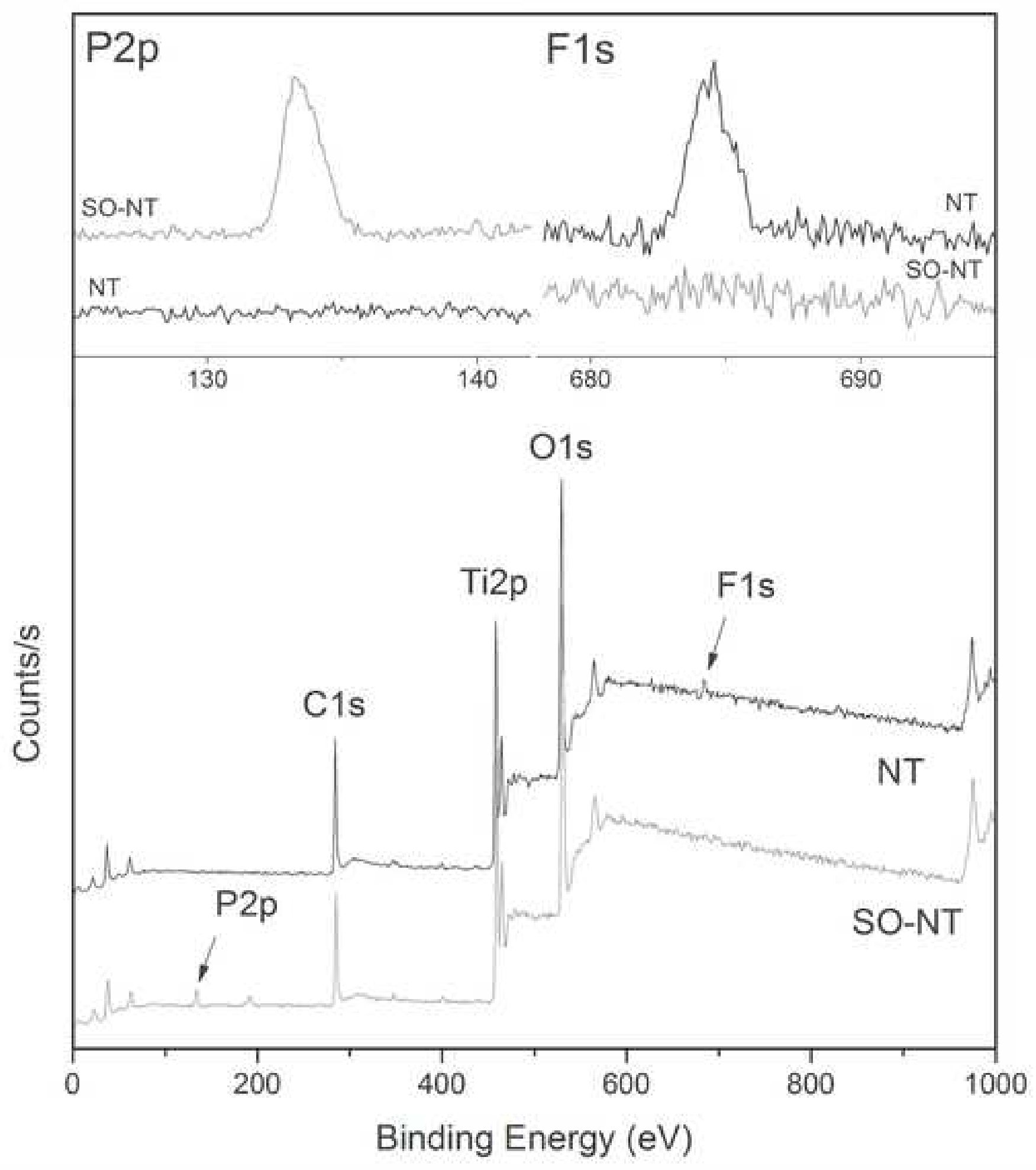


a
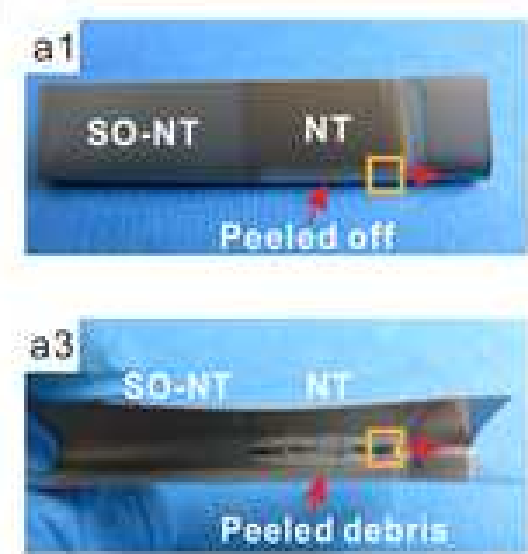

b

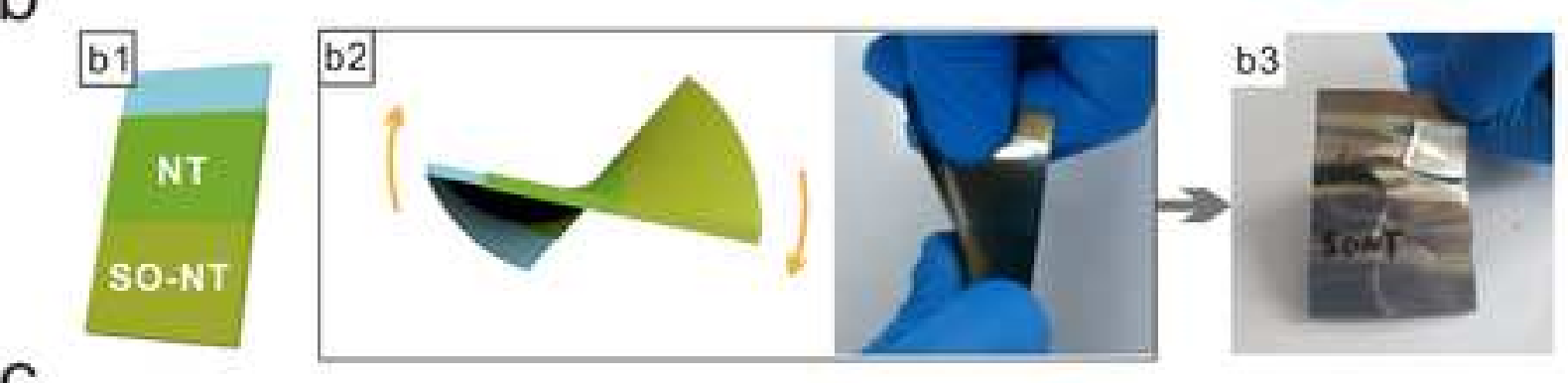

C

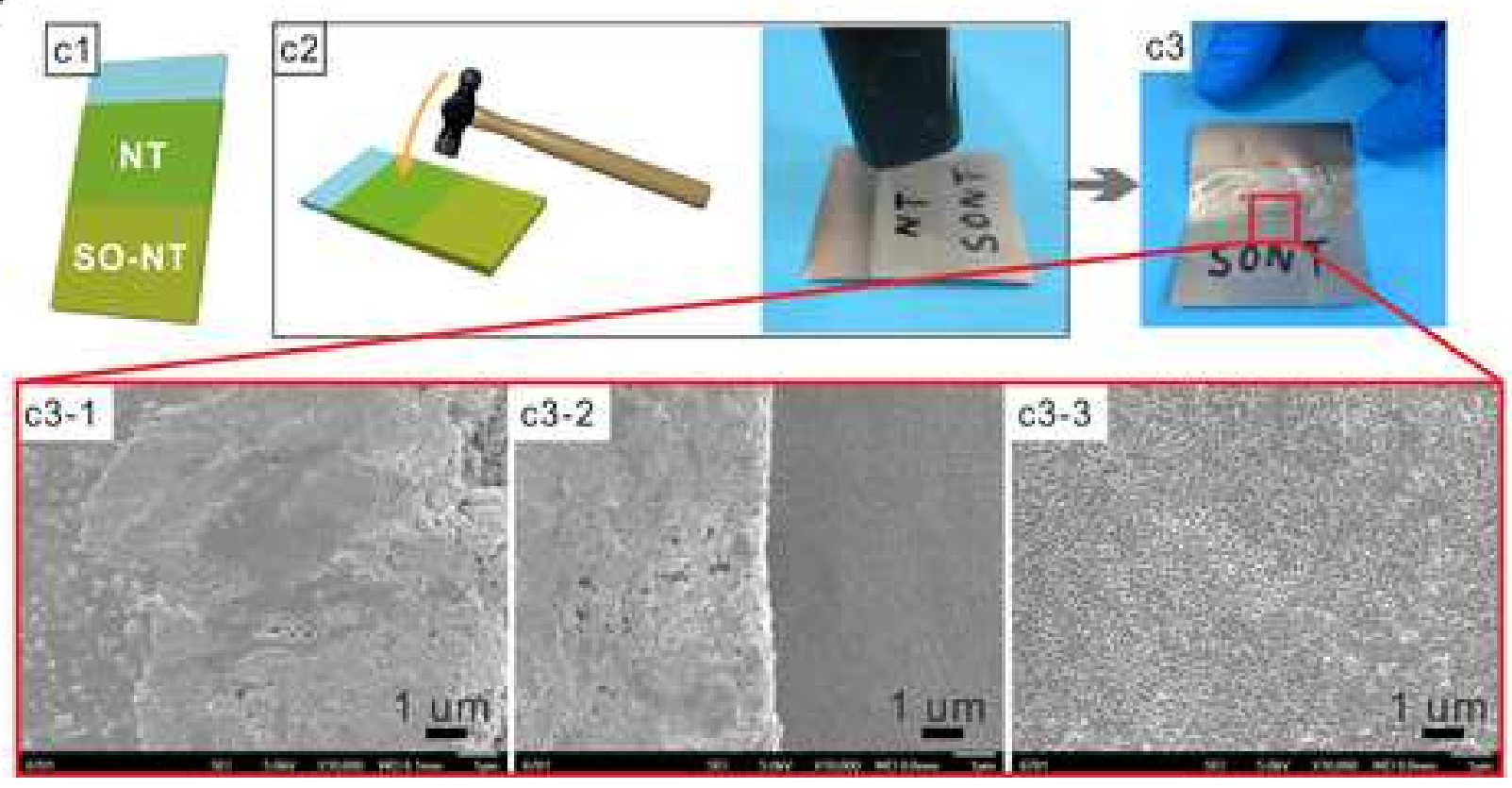

d

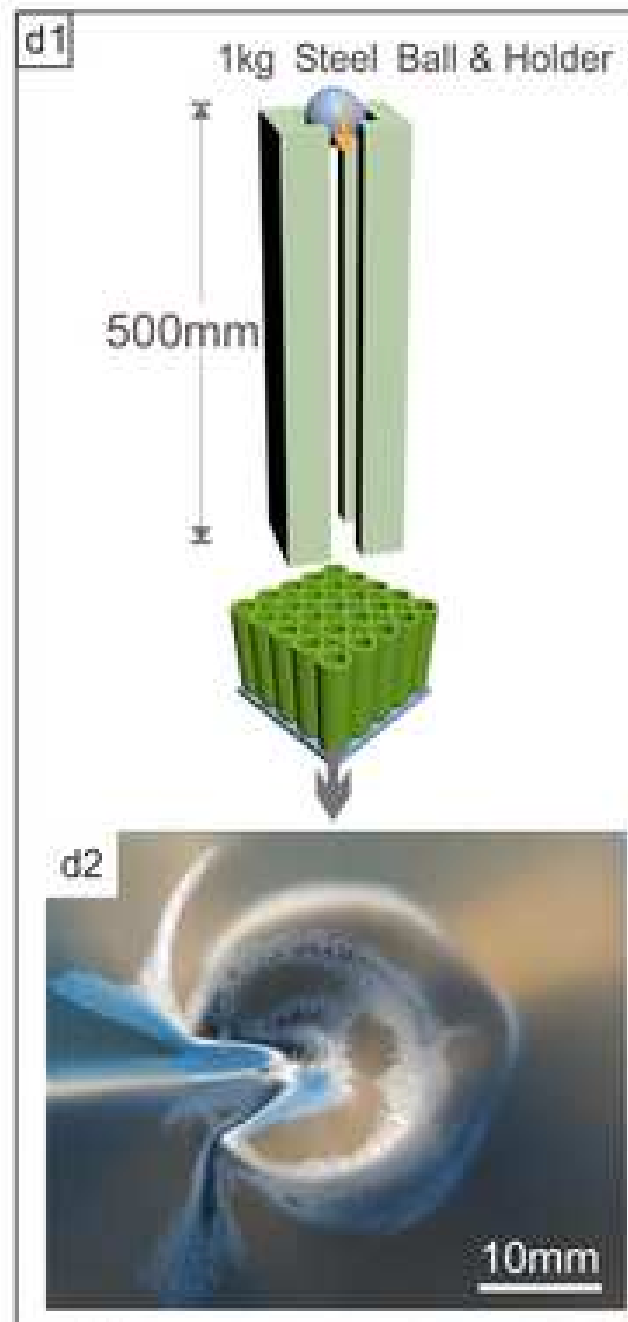

d3

$10 \mathrm{~mm}$ 


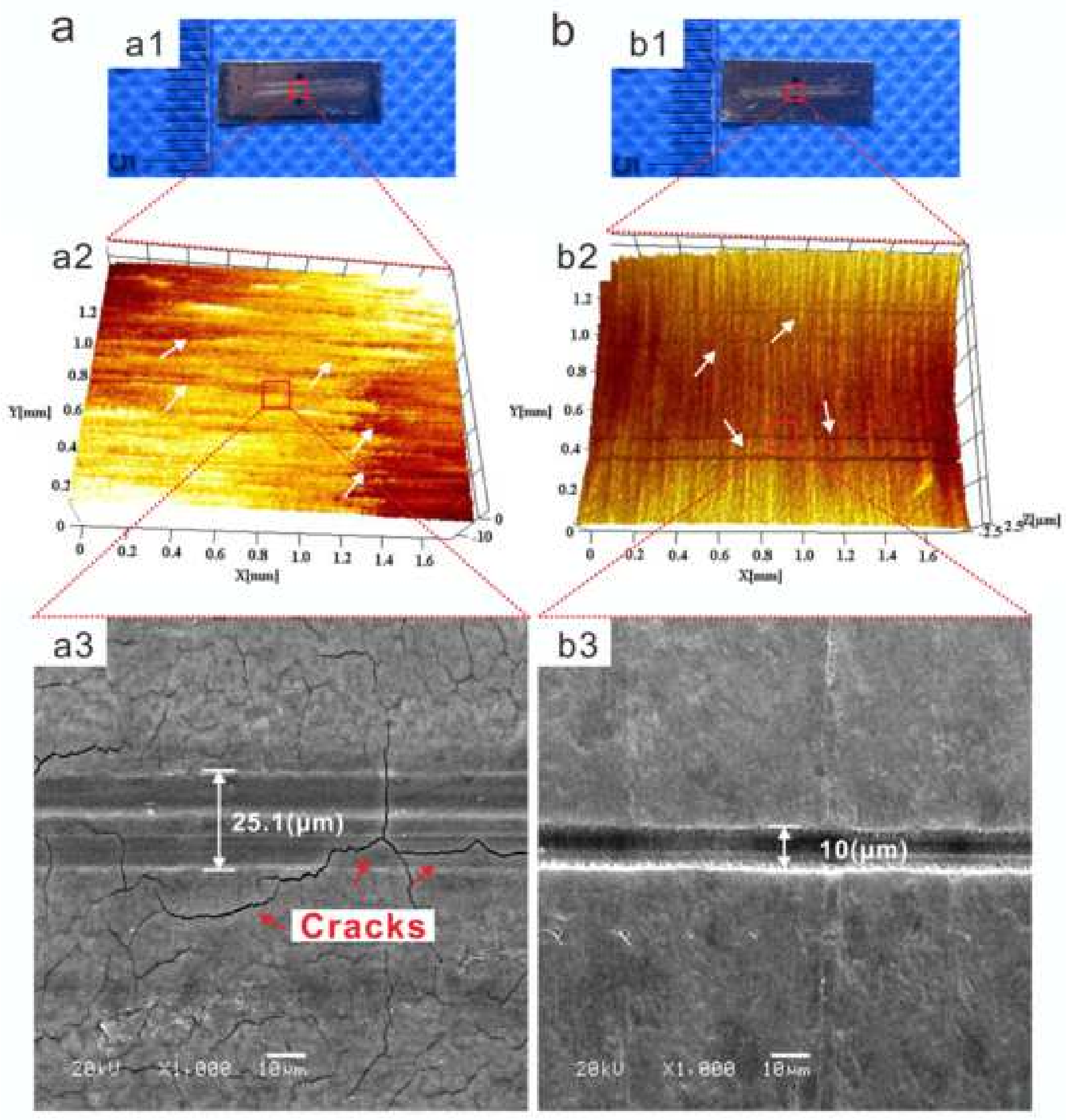




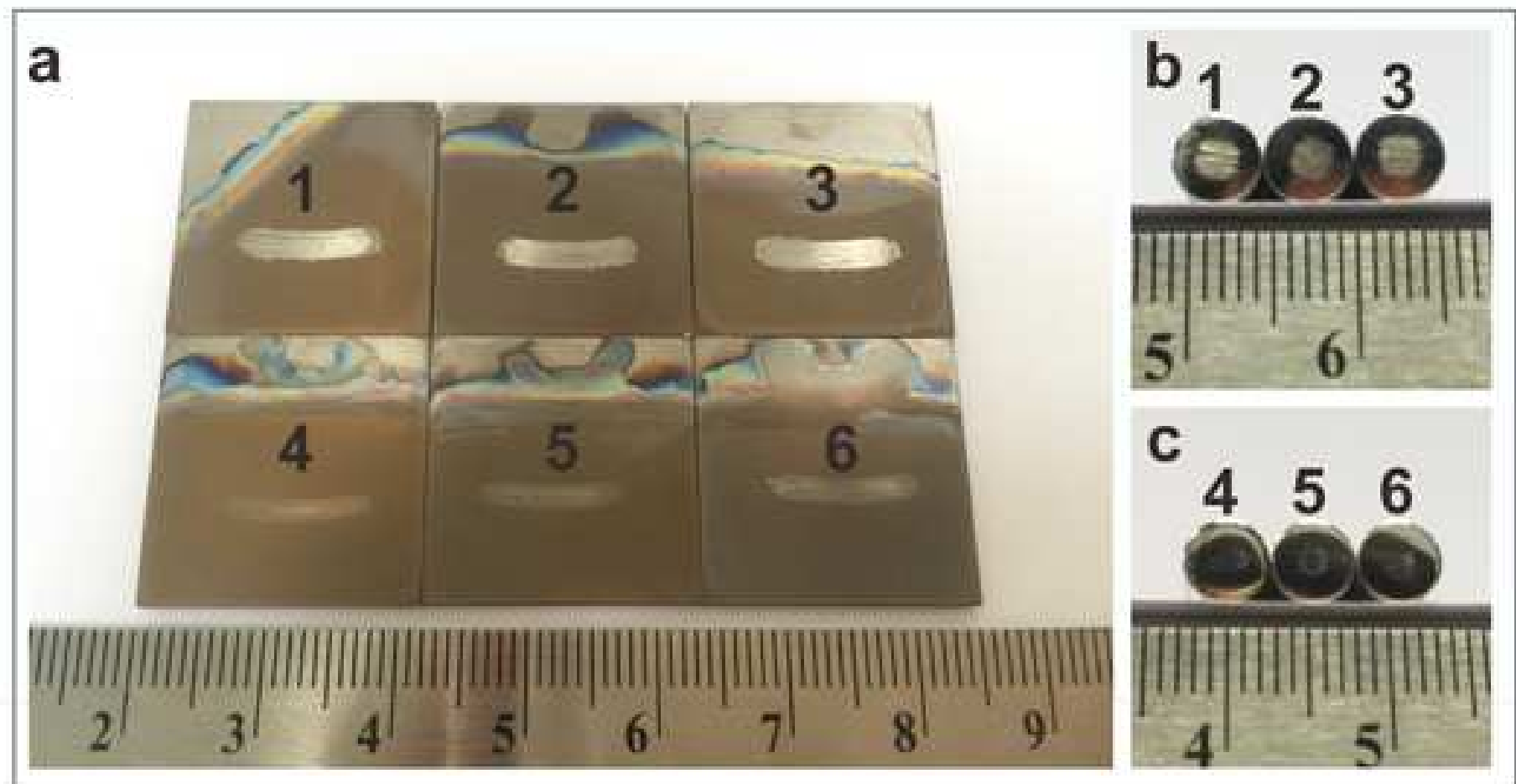

\section{c}

d 0.6

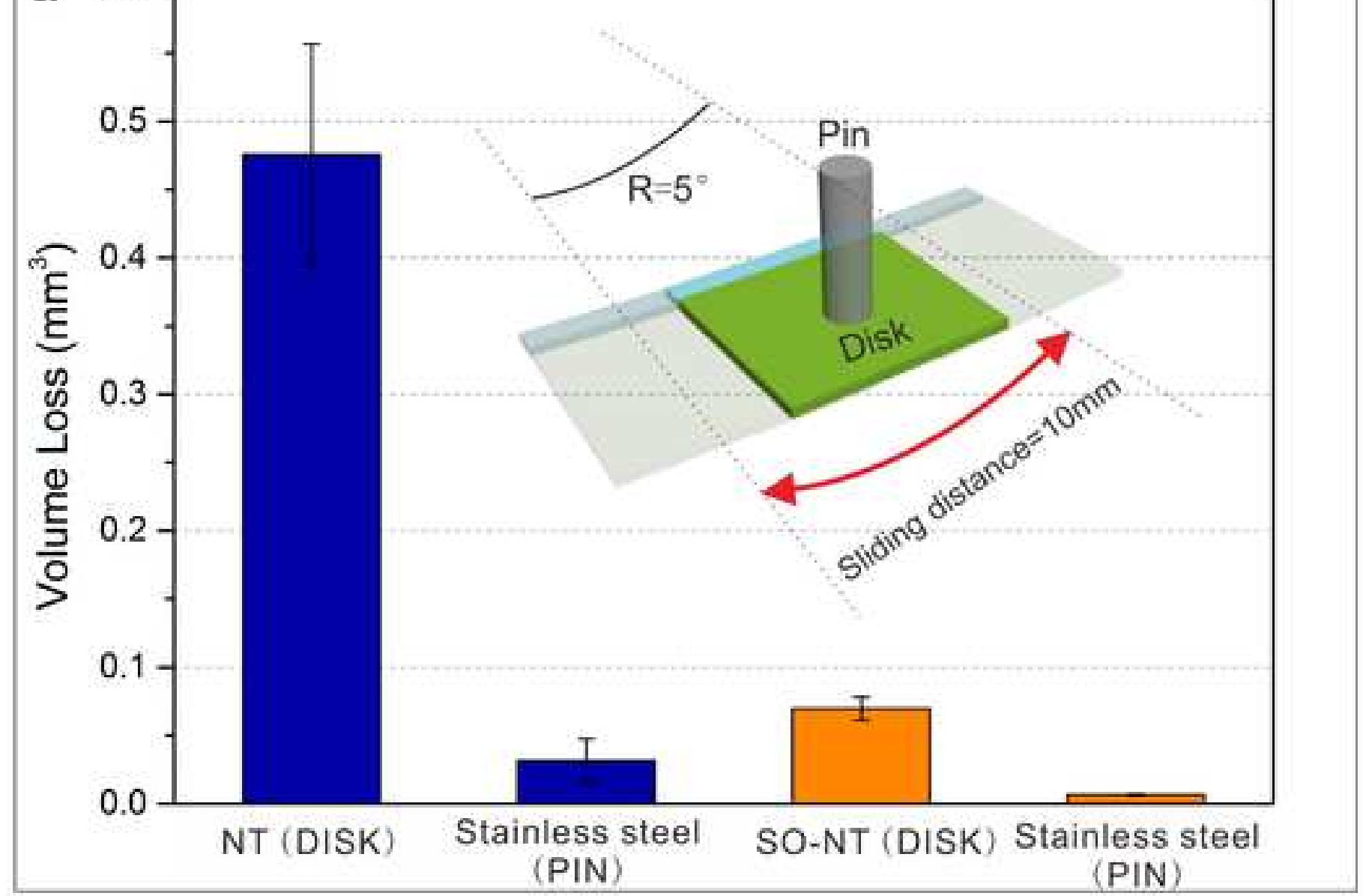




\section{a Deformation}

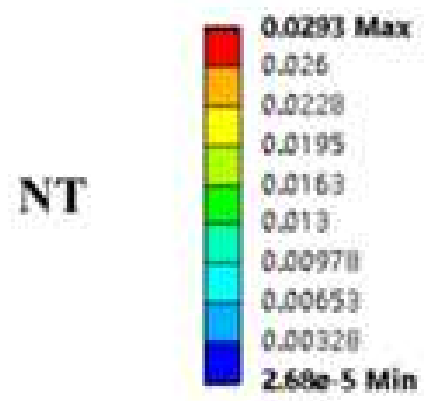

(aim)

\section{Deformation}

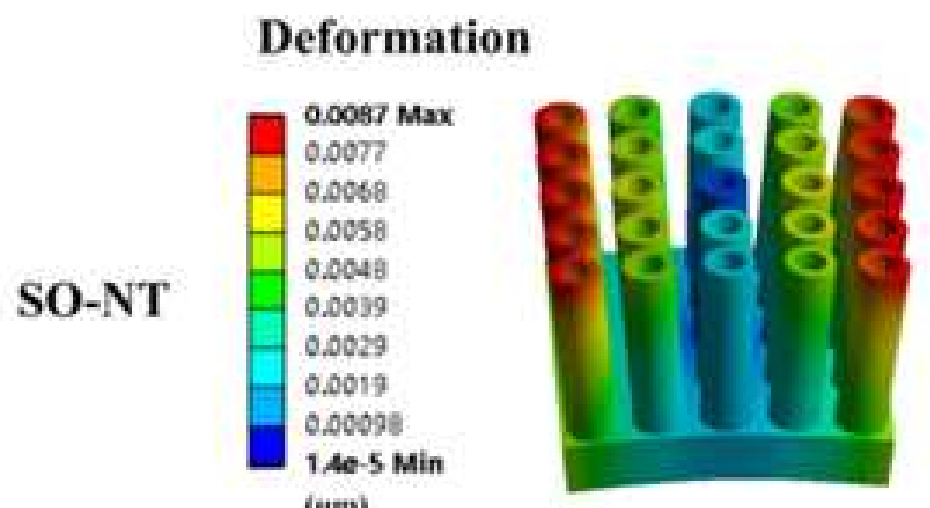

(нm)

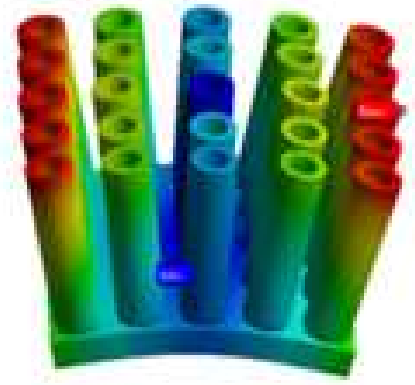

\section{Stress}

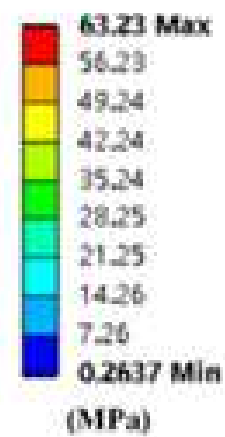

\section{Stress}

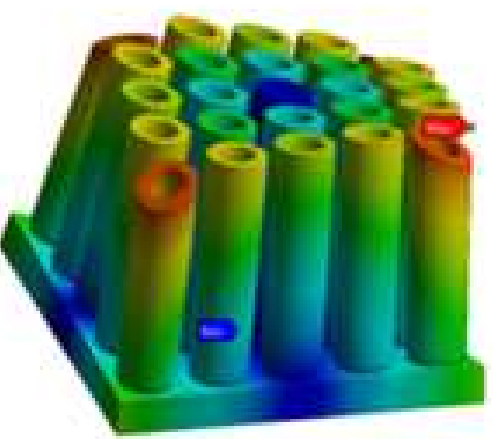

\section{Deformation}
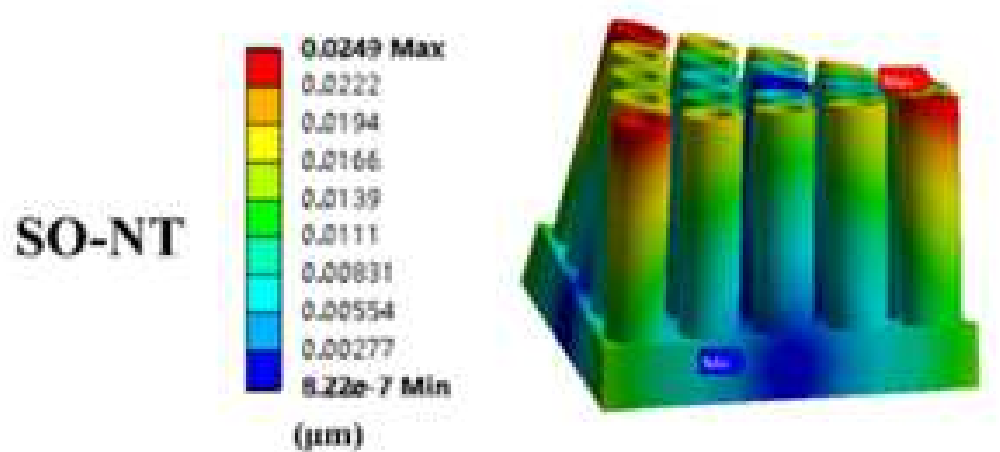

(MPa)

\section{Stress}

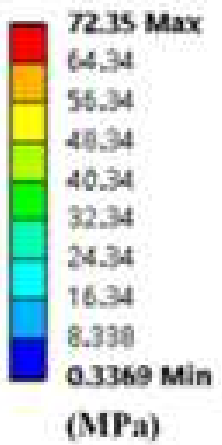

(MPa)
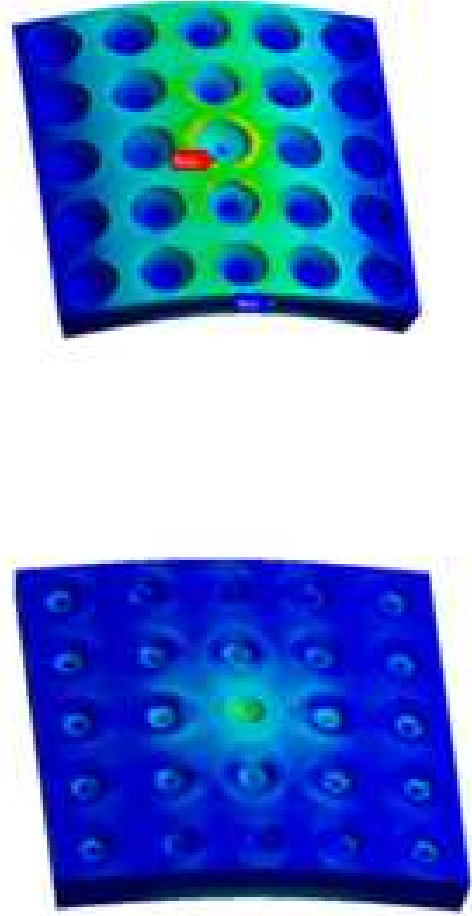
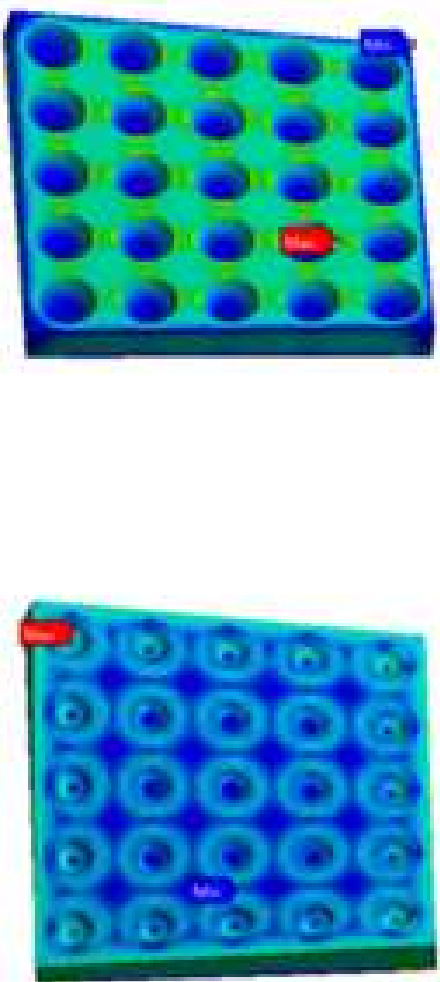
a

b
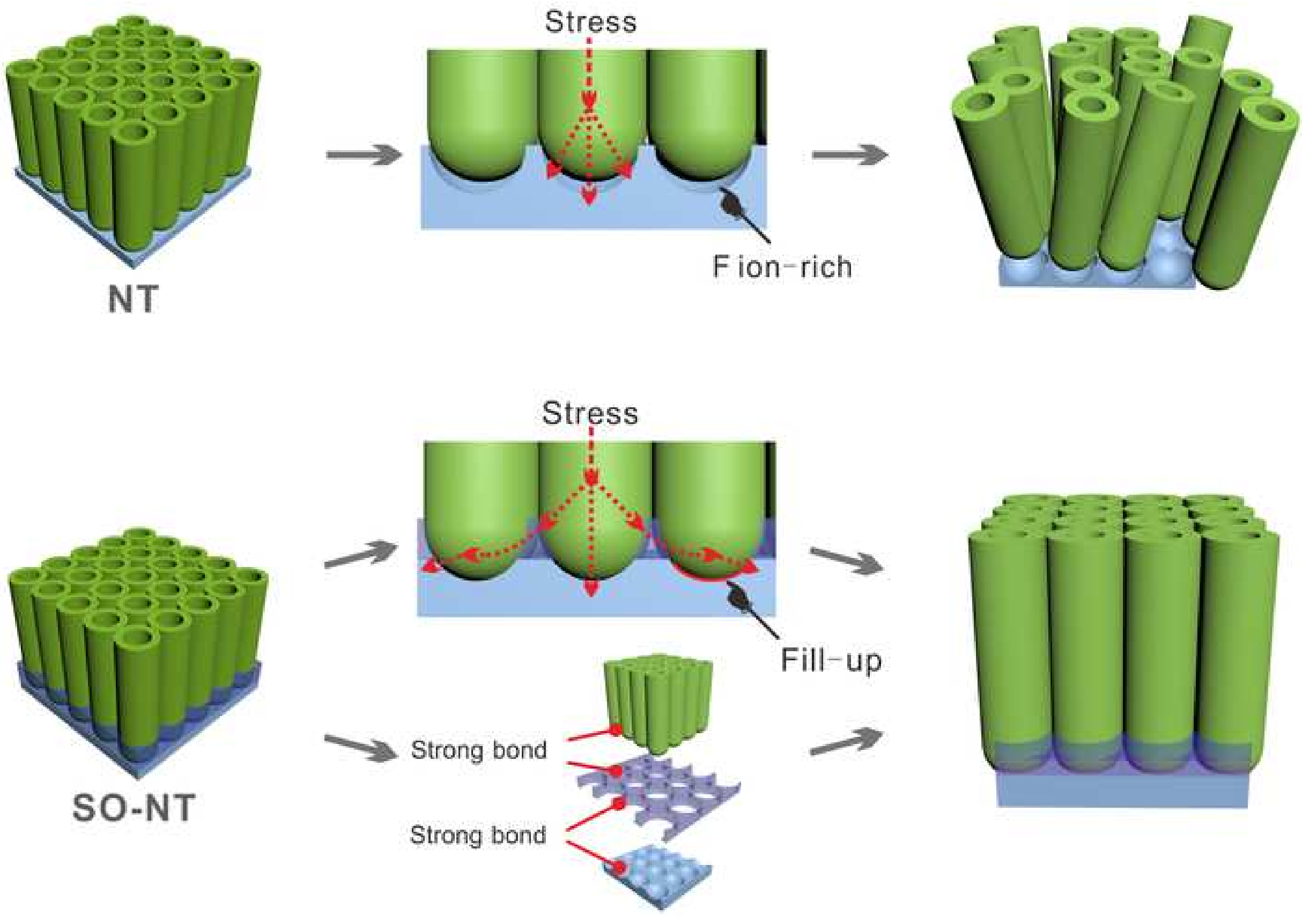

Fion-rich 


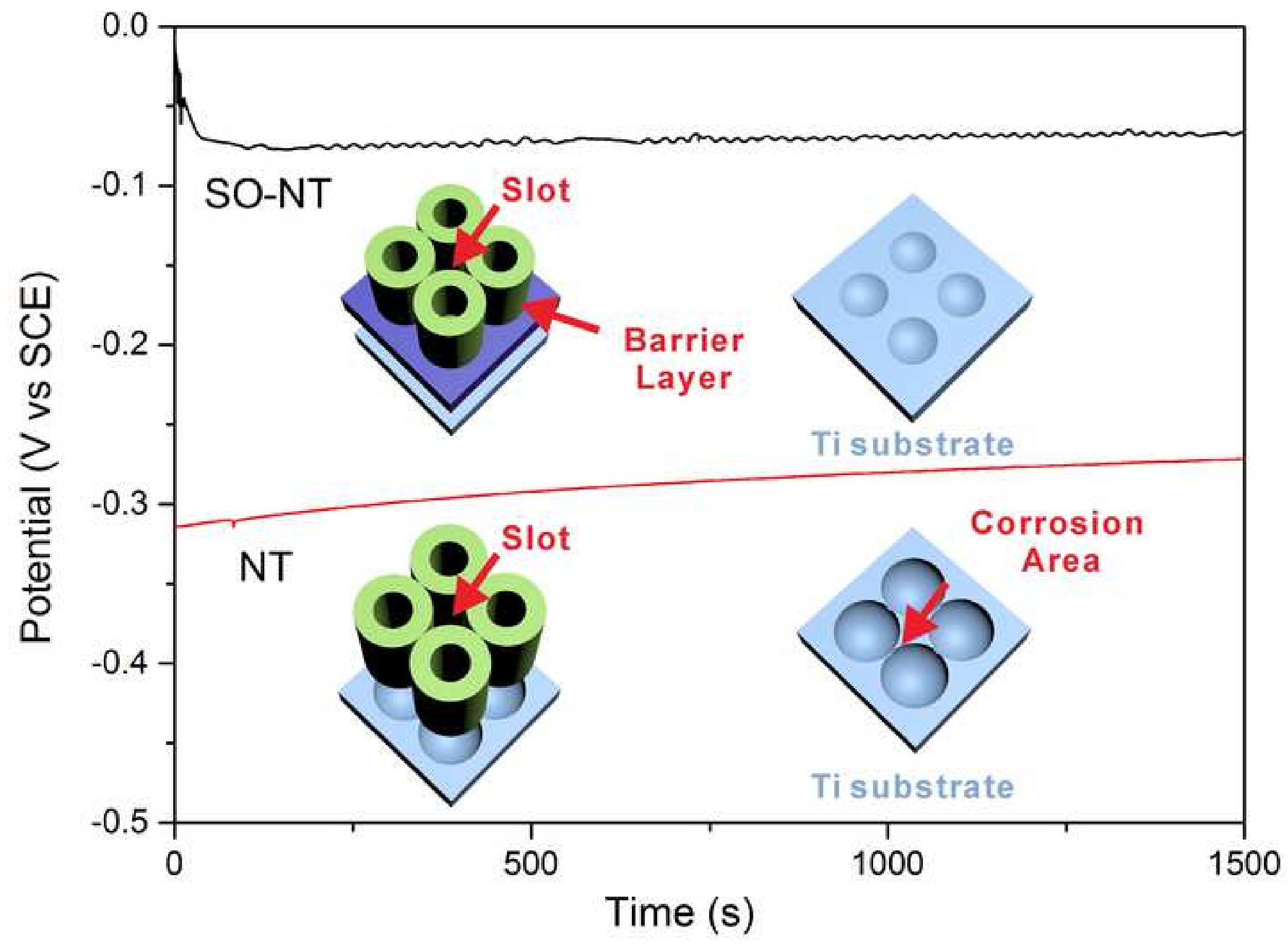




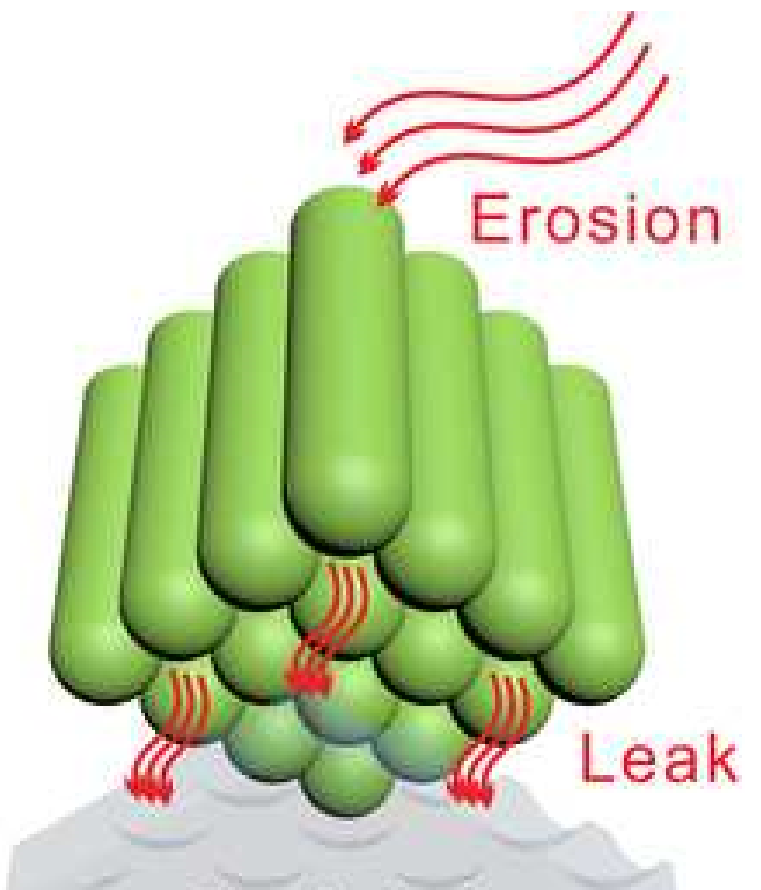

\section{Ti substrate}

NT

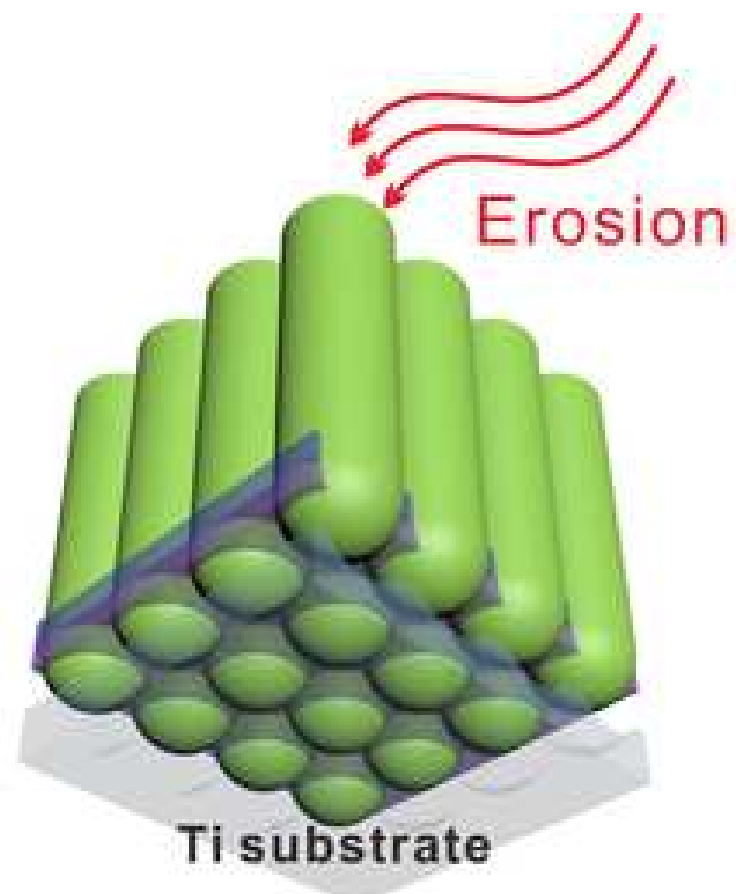

SO-NT 


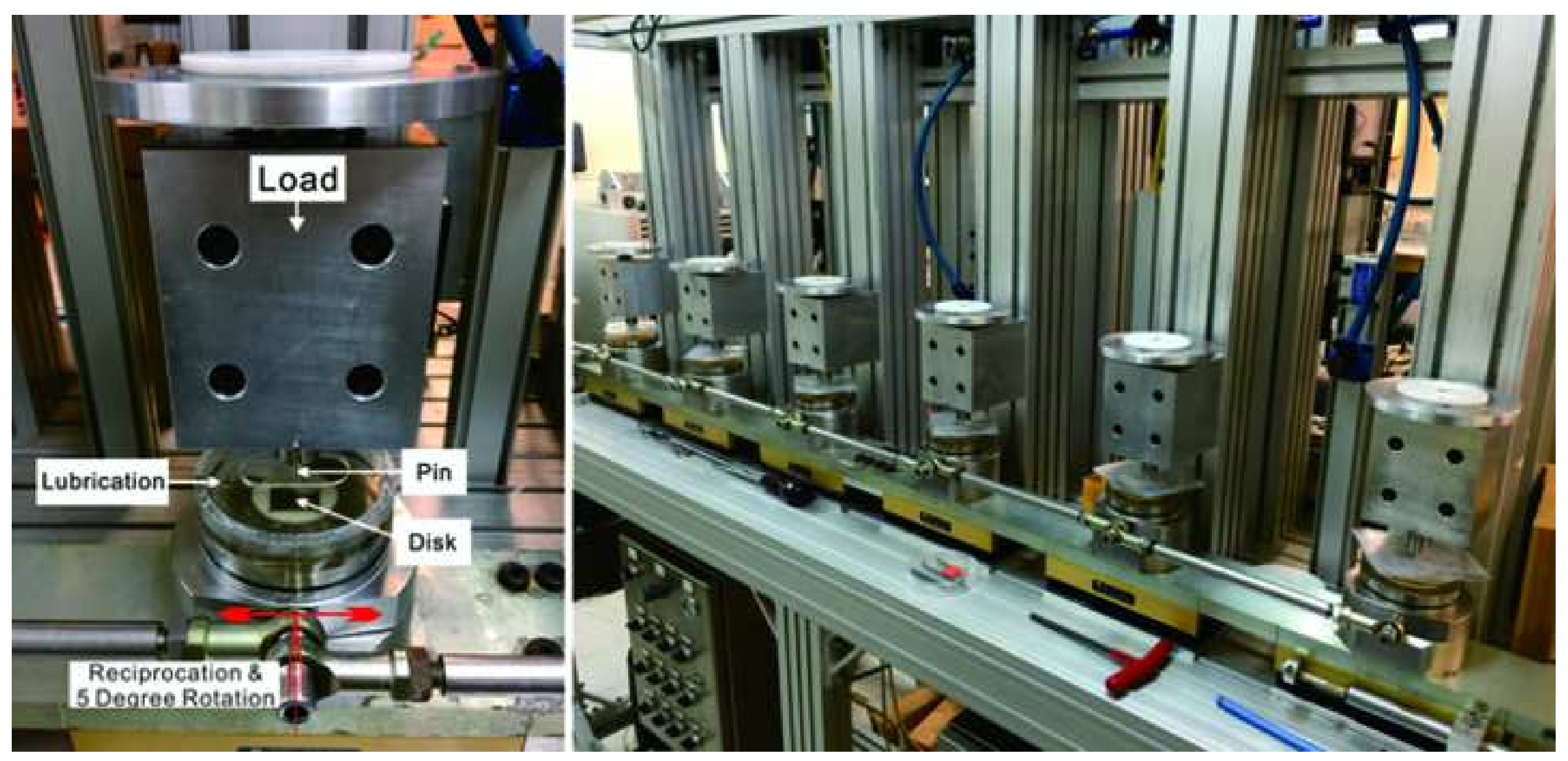




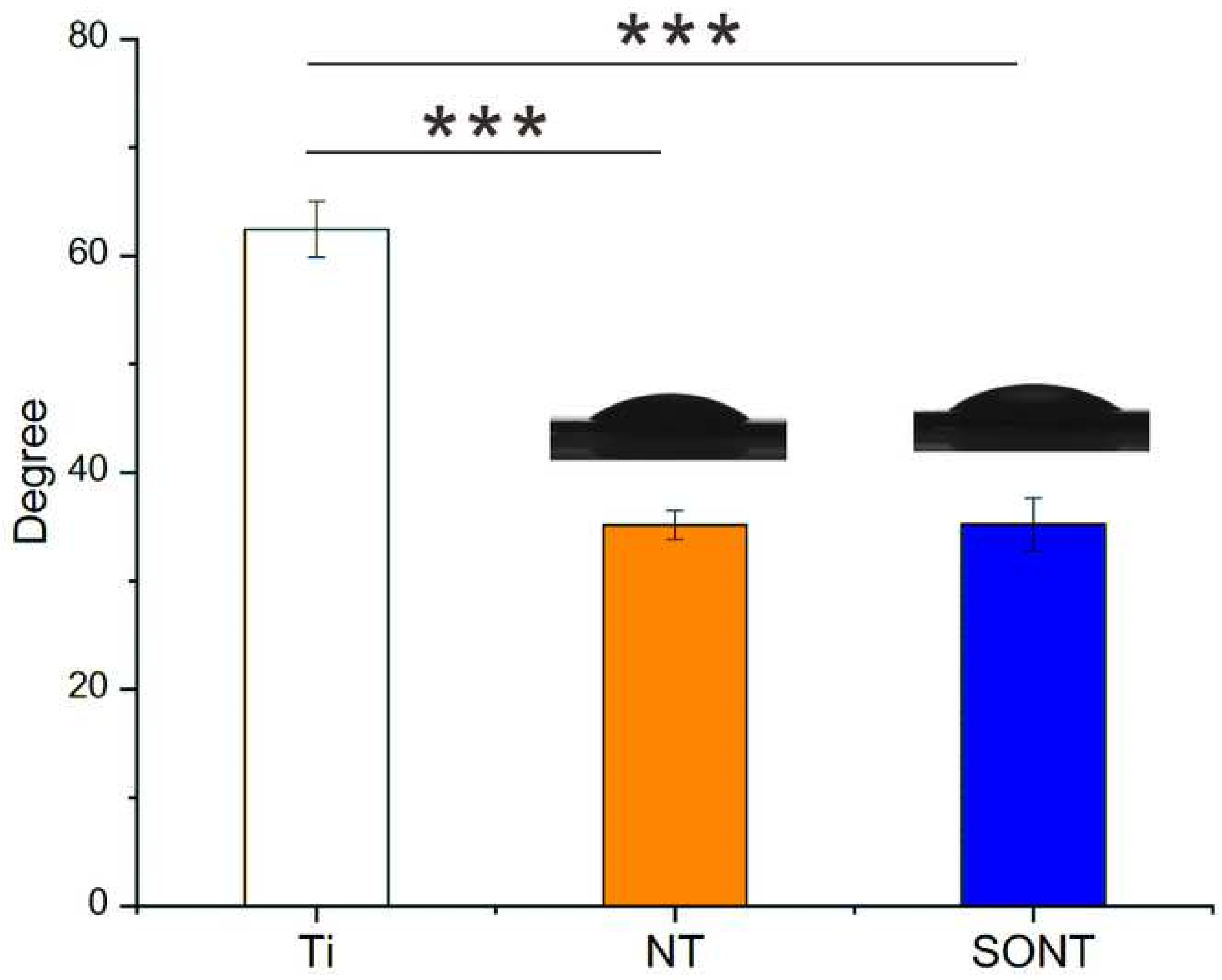




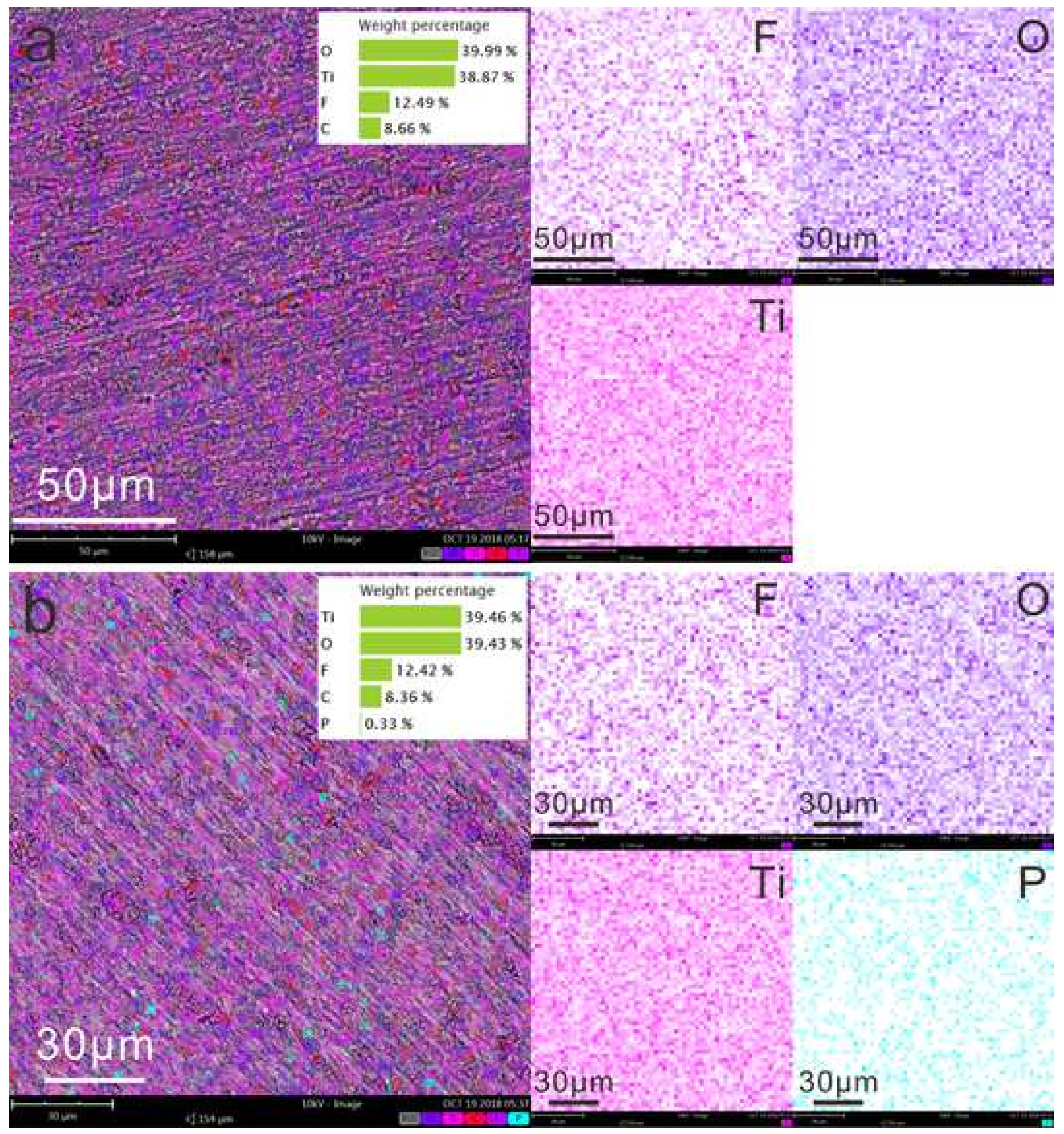



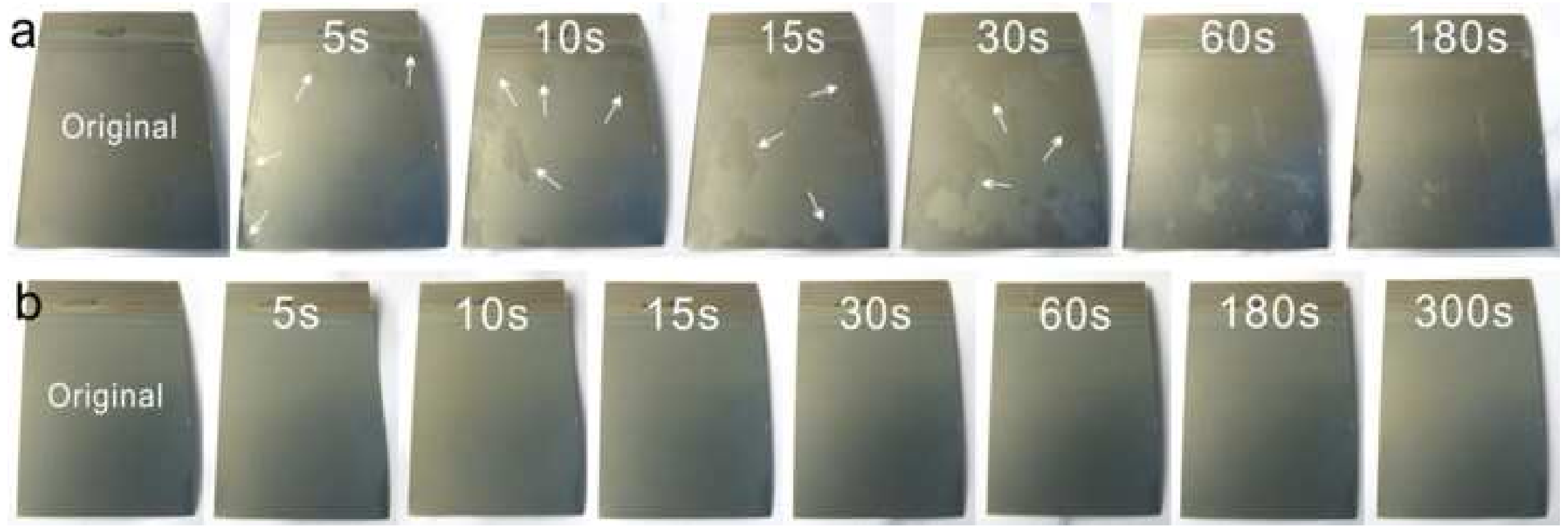


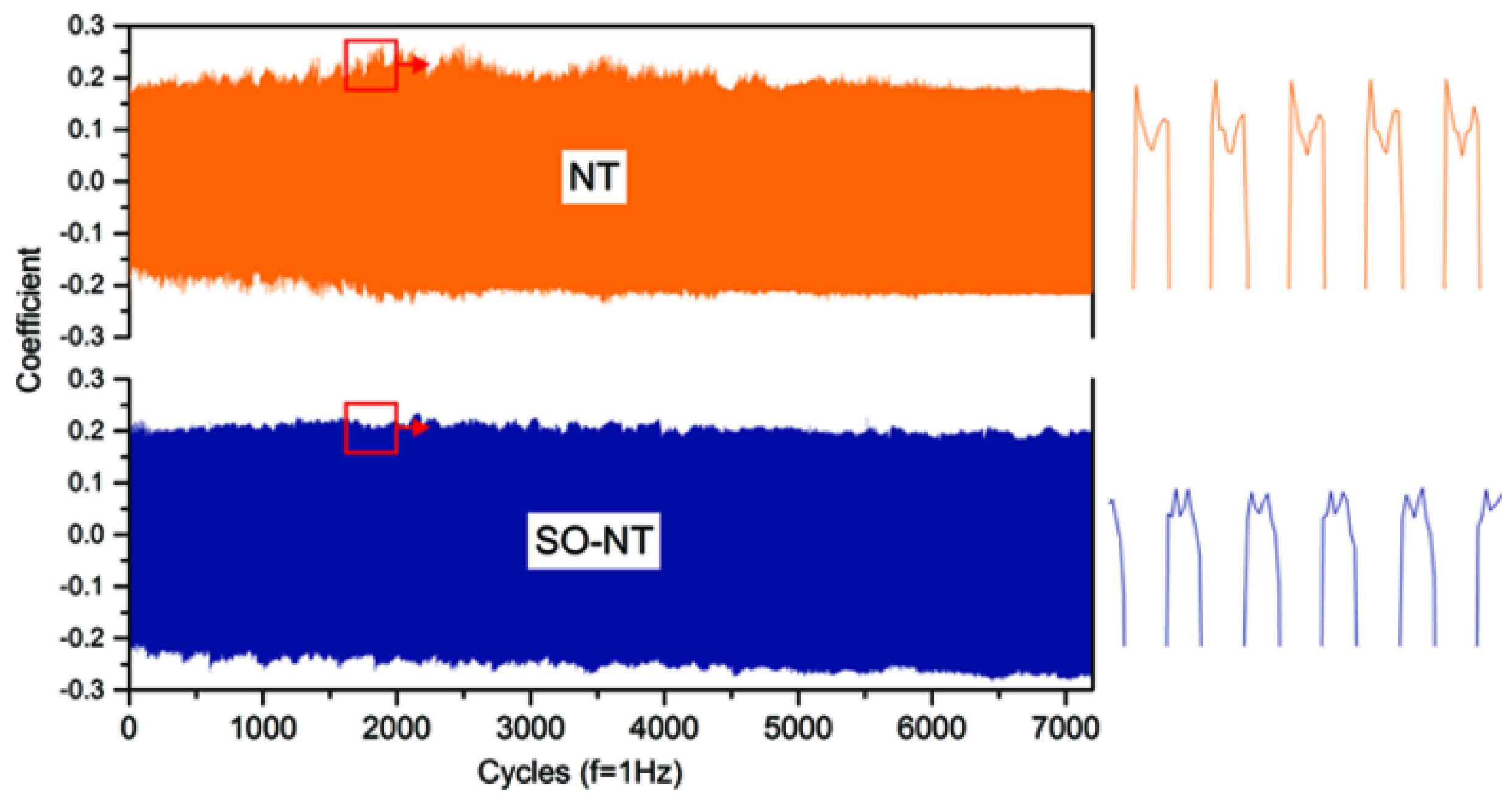




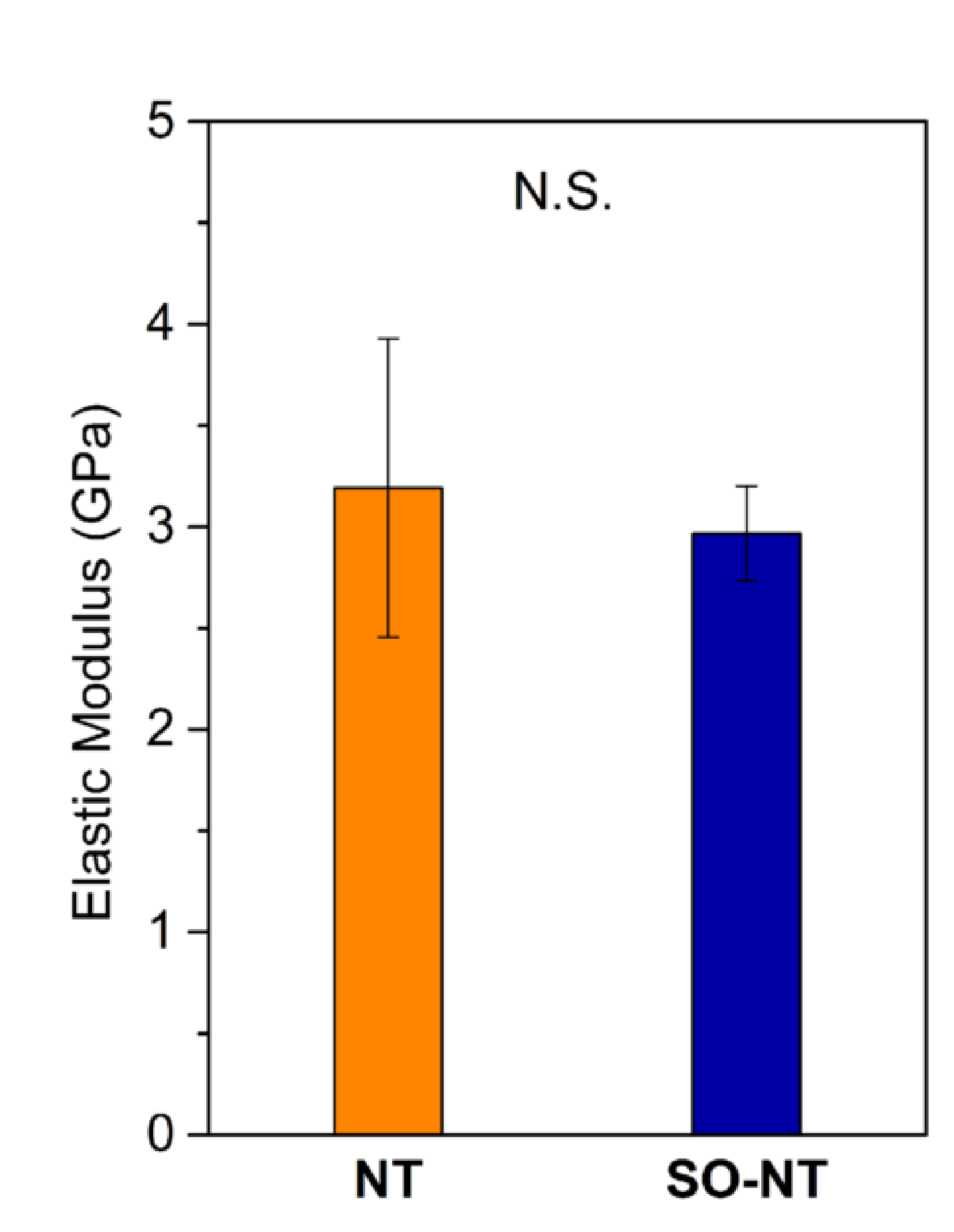

Figure S6

. 


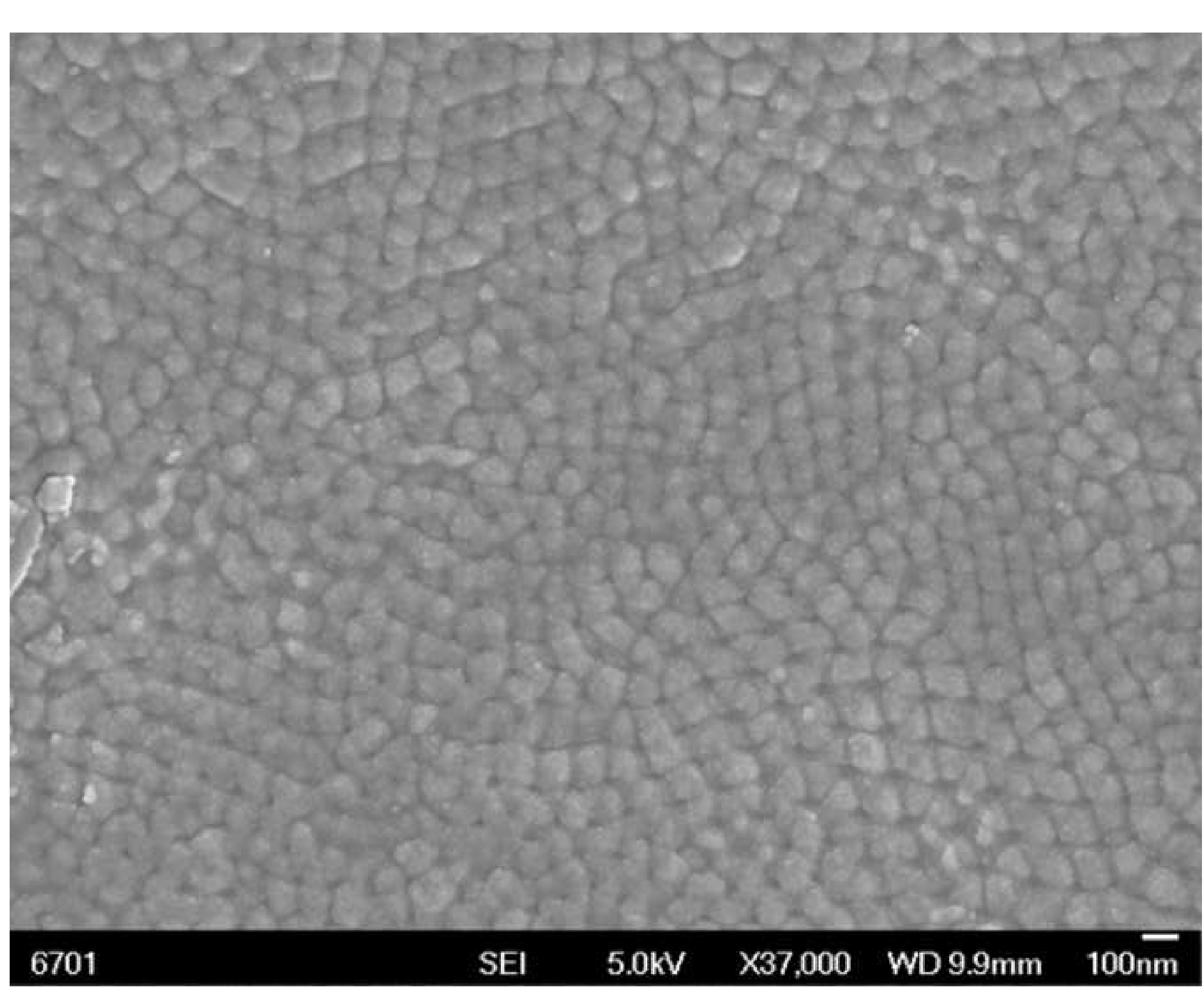




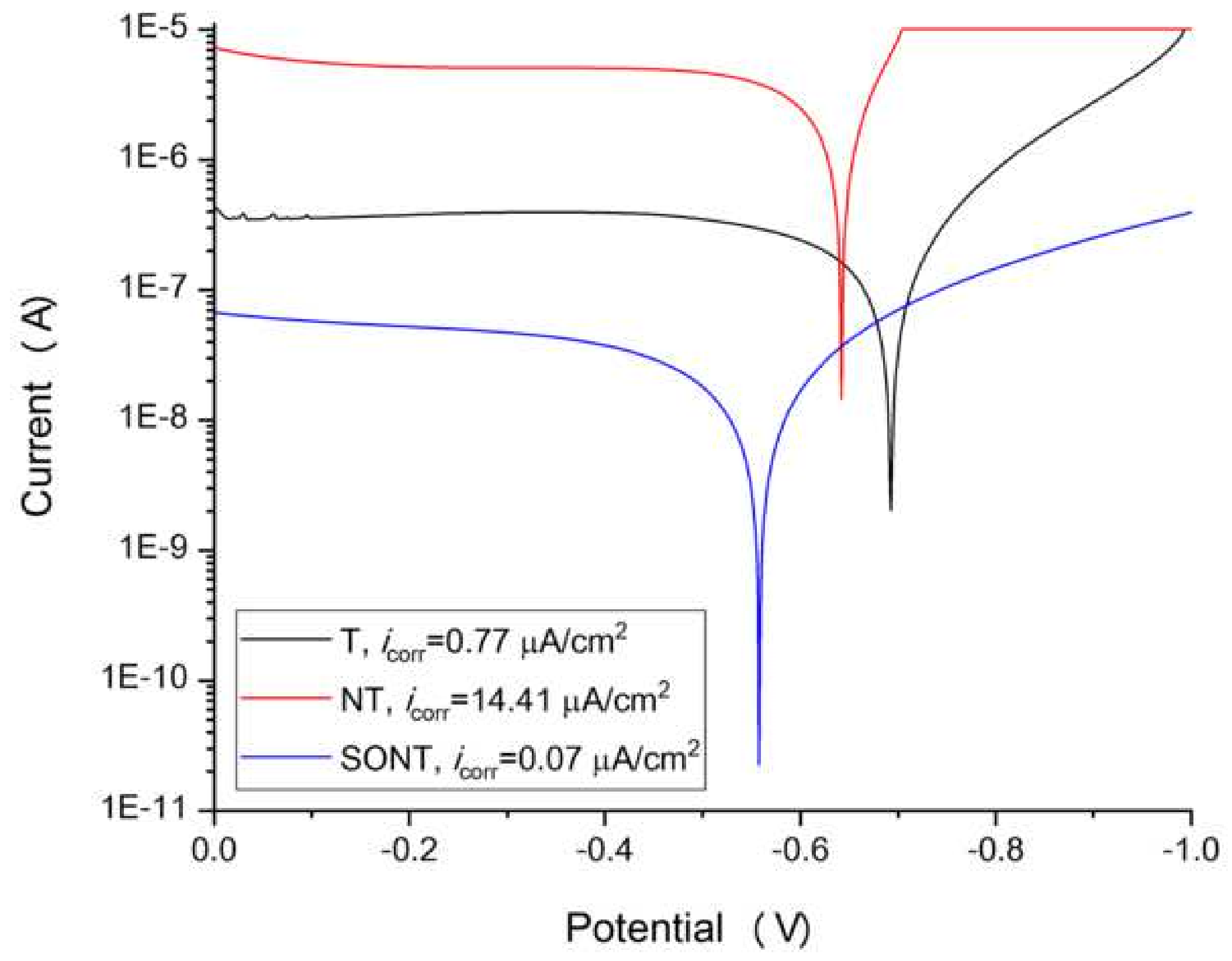


Click here to access/download Supplementary Material JBE_Supporting information-20191101.docx 